Management international

International Management

Gestiòn Internacional

\title{
Lieuité et socialisation organisationnelle : les raisons du lieu pour les travailleurs de très petites entreprises créatives et culturelles
}

\section{Placeness and Organisational Socialisation: Influences of Place for Employees of Creative and Cultural Micro-Enterprises \\ Lugar y socialización organizacional: razones de la localización de los trabajadores de pequeñas empresas creativas y culturales}

\author{
Anne-Laure Saives, Brigitte Charles-Pauvers, Nathalie Schieb-Bienfait et Basile \\ Michel
}

Volume 21, numéro 1, automne 2016

URI : https://id.erudit.org/iderudit/1052496ar

DOI : https://doi.org/10.7202/1052496ar

Aller au sommaire du numéro

Éditeur(s)

HEC Montréal

Université Paris Dauphine

ISSN

1206-1697 (imprimé)

1918-9222 (numérique)

Découvrir la revue

Citer cet article

Saives, A.-L., Charles-Pauvers, B., Schieb-Bienfait, N. \& Michel, B. (2016). Lieuité et socialisation organisationnelle : les raisons du lieu pour les travailleurs de très petites entreprises créatives et culturelles. Management international / International Management / Gestiòn Internacional, 21(1), 41-57.

https://doi.org/10.7202/1052496ar
Résumé de l'article

En quoi le lieu géographique, à l'échelle du quartier, participe-t-il de l'implication au travail pour des travailleurs spécifiques : les professionnels créatifs indépendants ou entrepreneurs salariés de TPE créatives et culturelles ? Nous appuyant sur la littérature récente en géographie relationnelle, nous examinons la « lieuité » dans le cas de ces travailleurs installés dans le quartier des Olivettes, un des « clusters créatifs » de la métropole nantaise. 32 entrevues individuelles révèlent deux séries de propriétés lieuitaires (identité, familiarité, vitalité et quotidienneté, centralité, connectivité et sérendipité) porteuses de socialisation organisationnelle. Le lieu-quartier apparaît comme une forme de tiers-organisation réductrice d'incertitude et d'instabilité.
Tous droits réservés (C) Management international / International Management / Gestión Internacional, 2016
Ce document est protégé par la loi sur le droit d'auteur. L’utilisation des services d’Érudit (y compris la reproduction) est assujettie à sa politique d'utilisation que vous pouvez consulter en ligne.

https://apropos.erudit.org/fr/usagers/politique-dutilisation/ 


\title{
Lieuité et socialisation organisationnelle : les raisons du lieu pour les travailleurs de très petites entreprises créatives et culturelles*
}

\author{
Placeness and Organisational Socialisation: Influences \\ of Place for Employees of Creative and Cultural \\ Micro-Enterprises
}

\section{Lugar y socialización organizacional: razones de la localización de los trabajadores de pequeñas empresas creativas y culturales}

\author{
ANNE-LAURE SAIVES BRIGITTE CHARLES-PAUVERS NATHALIE SCHIEB-BIENFAIT BASILE MICHEL
ESG-UQAM, Montréal, Canada
IEMN-IAE, LEMNA,
Université de Nantes, France
IEMN-IAE, LEMNA,
Université de Nantes, France
Laboratoire ESO-Angers
UMR CNRS 6590,
et Université d'Angers, France

\section{RÉSUMÉ}

En quoi le lieu géographique, à l'échelle du quartier, participe-t-il de l'implication au travail pour des travailleurs spécifiques : les professionnels créatifs indépendants ou entrepreneurs salariés de TPE créatives et culturelles? Nous appuyant sur la littérature récente en géographie relationnelle, nous examinons la "lieuité» dans le cas de ces travailleurs installés dans le quartier des Olivettes, un des "clusters créatifs" de la métropole nantaise. 32 entrevues individuelles révèlent deux séries de propriétés lieuitaires (identité, familiarité, vitalité et quotidienneté, centralité, connectivité et sérendipité) porteuses de socialisation organisationnelle. Le lieu-quartier apparaît comme une forme de tiers-organisation réductrice d'incertitude et d'instabilité.

Mots clés : Lieu, lieuité, entrepreneurs salariés, travailleurs indépendants, activités créatives et culturelles, socialisation organisationnelle, implication au travail, quartier

\section{ABSTRACT}

In what way does geographic location, at the neighbourhood level, influences the work commitment of a specific set of workers: independent creative professionals or salaried entrepreneurs in creative, cultural microenterprises? Referring to recent literature on relational geography, we examine "placeness" in the context of workers in the "Quartier des Olivettes”, one of Nantes Metropole's creative clusters. Thirty-two individual interviews reveal two series of placeness properties (identity, familiarity, vitality and everydayness; centrality, connectivity and serendipity) which support organisational socialisation. The place/quarter emerges as a third-organisation reducing incertitude and instability

Keywords: Place, placeness, salaried entrepreneurs, freelancers, creative and cultural activities, organisational socialisation, work commitment, quarter

\section{RESUMEN}

En qué medida el lugar geográfico, a la escala del barrio, afecta al trabajo de los creadores independientes y de los empresarios de pequeñas empresas creativas y culturales? Inspirados por la reciente literatura sobre la geografía relacional, investigamos el rol del lugar geográfico en las prácticas y actitudes de los trabajadores del barrio de Olivettes, una aglomeración creativa de la ciudad de Nantes. 32 entrevistas individuales revelan la importancia de dos series de propiedades del lugar (identidad, familiaridad, vitalidad y cotidianidad, centralizad, conectividad y serendipia) portadoras de socialización organizacional. El barrio aparece así como una forma de organización-tercera reductora de la incertidumbre y de la inestabilidad.

Palabras clave: Lugar, localización, empresarios, trabajadores independientes, actividades creativas y culturales, socialización organizacional, implicación laboral, barrio
$\mathrm{D}$ epuis, entre autres, les publications de Caves (2000) sur les industries créatives et de Florida sur la "classe créative» (2002, 2004, 2005), les politiques publiques de certaines métro- poles et grandes villes se sont attachées au concept de cluster ${ }^{1}$ pour reproduire le modèle des grappes industrielles, emprunté à Porter (1998), aux industries créatives et culturelles ${ }^{2}$ dans la quête

\footnotetext{
* Cette recherche s’inscrit dans le cadre du programme de recherche VALEUR(S) et Utilités de la culture, financée par la région Pays de la Loire. Dans le cadre de cette recherche, un autre article a été réalisé sur l'ensemble des entreprises créatives implantées dans ce quartier. Nous tenons à remercier Zoé Wambergue. 1. M. Porter (1998) définit un cluster comme un groupe d'entreprises et d'institutions partageant un même domaine de compétences, proches géographiquement, reliées entre elles et complémentaires.

2. Cette notion "d'industrie créative» renvoie aux activités artistiques et aux industries culturelles (architecture, cinéma, édition, vidéo, audiovisuel, photographie, musique, spectacle vivant) tout en justifiant l'extension des règles de protection de la propriété intellectuelle à de nouveaux secteurs (publicité, design, mode, jeux vidéo, internet, logiciel) (Tremblay, 2008).
} 
d'une redynamisation de leur tissu économique par la création d'activité et d'emplois dans ces secteurs (Hall, 2000; Turok, 2003; Smith et Warfield, 2007; Bayliss, 2007; Cooke et Lazzerreti, 2008; Aage et Belussi, 2008; Scott, 2010a, 2010b; Liefooghe, 2010). Une littérature aujourd'hui foisonnante s'interroge sur la place et le rôle de la classe créative (Clifton et Cooke, 2009) dans la dynamique de clusterisation (Ambrosino et Andres, 2007; Ambrosino, 2009; Chapain et al., 2010; Comunian et al., 2010; Andres et Chapain, 2013). Toutefois, la réalité située du travail des entrepreneurs de ces secteurs créatifs et culturels à une échelle infra-métropolitaine ne semble pas véritablement connue (Clifton and Cooke, 2009; Chapain et al., 2013) ni prise en compte dans ces politiques «top down». Les conditions de travail de ces entrepreneurs, porteurs de projet d'activité indépendante et/ou acteurs d'organisations de très petite taille, invitent à revisiter les leviers d'intégration économique et sociale.

Constatant la concentration de travailleurs dits "créatifs» (Vivant, 2011) dans un quartier populaire de la ville de Nantes, le quartier des Olivettes, nous souhaitons examiner le lien entre le lieu géographique du travail et l'intégration-implication au travail (ou 'work commitment', Morrow, 1993); le travail ou «work» recouvre ainsi la «profession », la «carrière», le «job" ou encore le «boulot» ainsi que l'organisation (Morrow, 1993; Allen et Meyer, 1996). En effet, l'idée d'intégration organisationnelle pose problème quand l'organisation de ce travailleur quel que soit son statu ${ }^{3}{ }^{3}$ 'existe pas à proprement parler. Nous faisons l'hypothèse que, confronté à une individualisation forte du rapport au travail, ainsi qu'à un fort isolement, le travailleur «dit créatif» vit singulièrement sa situation alors qu'il est face à lui-même à longueur de journée. La question se pose de savoir en quoi et à quoi il se sent intégré. De fait, nous nous demandons en quoi le lieu (ici le quartier) contribue-t-il à l'implication au travail (et plus largement au sens du travail) et peut-il tenir place de quasi-organisation?

Comme le souligne E. Vivant (2009), bien que ces «travailleurs créatifs» aient un lien fort à l'espace urbain et que leurs conditions de précarité et la flexibilisation de leurs emplois tendent à se diffuser à l'ensemble du salariat, peu de travaux s'intéressent aux problématiques soulevées par ces formes d'organisations sociales ou spatiales spécifiques. Notre objectif est de réinvestir le concept de lieu géographique de travail ici le quartier - en tant qu'espace et place pour le comprendre comme une forme d'organisation spécifique, avec laquelle la plupart des travailleurs dits créatifs auraient créé une relation. Notre question devient alors : comment définir ce lieu? Ou encore quelles sont ses caractéristiques qui participent de l'intégration-implication au travail et de la production de sens pour ces travailleurs spécifiques (Menger, 2005; Vivant, 2011)?

Croisant la littérature récente en géographie relationnelle et sociale (Massey, 1991, 2005; Cresswell, 2007) en particulier sur les concepts de 'place/space', de 'global sense of place' et sur la «lieuité» (Lévy et Lussault, 2003), avec la littérature sur l'intégration et l'implication au travail (Klein et al., 2012;
Vandenberghe, 2009), nous tenterons de questionner et de comprendre les raisons de l'ancrage «lieuitaire» de travailleurs dits créatifs souvent autonomes à partir d'un cas concret : les artistes et professions créatrices (architectes, designers web, publicitaires, chargés de communication, etc.) du quartier des Olivettes à Nantes, un des lieux désignés comme un "cluster créatif» par les responsables politiques de la métropole nantaise.

Le papier s'organise donc en trois temps : 1) présentation du cadre théorique articulant une compréhension du travailleur dit créatif, une caractérisation de la lieuité (caractéristiques qui «font» lieu) ainsi qu’une proposition de définition del'intégration et de l'implication au travail; 2) présentation du terrain nantais, du quartier et des très petites entreprises (TPE) créatives et culturelles étudiées; 3 ) présentation, analyse et discussion des résultats de l'enquête qualitative exploratoire menée auprès des travailleurs du quartier des Olivettes

\section{Cadrage théorique}

\section{LE TRAVAILLEUR-DIT-CRÉATIF : UN TRAVAILLEUR AU PROJET EN DEMANDE D'ORGANISATION?}

Les figures de l'artiste et du professionnel créatif se mêlent aujourd'hui dans la catégorie du «travailleur créatif». Selon Hillaire (2011), s'affirmerait l'idée qu'aujourd'hui l'artiste et le travailleur des temps post-industriels fusionneraient dans une seule et même figure, celle du «créatif». De plus, Menger (2002, p. 8) constate que «les activités de création artistiques ne sont pas ou plus l'envers du travail, mais (...) sont au contraire de plus en plus revendiquées comme l'expression la plus avancée des nouveaux modes de production et des nouvelles relations d'emploi engendrés par les mutations récentes du capitalisme». Selon E. Vivant $(2006,2011)$, les travailleurs sont plus largement dits créatifs parce qu'ils travaillent dans les industries créatives. Ces industries «trouvent leur origine dans la créativité, la compétence et le talent individuels, et ont un potentiel pour créer de la richesse et des emplois à travers la génération et l'exploitation de la propriété intellectuelle» (DCMS, 2001). Pour autant, ces travailleurs ne constituent pas une catégorie homogène. Comme le soulignent les travaux développés par $\mathrm{SMartBe}^{4}$ en 2011, l'artiste, quelle que soit sa discipline, peut être considéré comme un entrepreneur dans la mesure où il est à la fois «travailleur au projet, créateur de son propre emploi, chef d'équipe quand il doit réunir autour de lui des collaborateurs aux talents divers, agent de son propre succès commercial ou médiatique, inventeur de structures nouvelles...» (p. 12). De Heusch et al. (2011) constatent que ces travailleurs «cumulent la discontinuité, la variabilité et la disponibilité du salarié et de l'entrepreneur, tout en refusant de s'identifier à l'un comme à l'autre» (Corsani et Lazzarato, 2008, p 78-79). Ils en cumulent les différentes fonctions et compétences, tantôt porteurs de projet, tantôt parties prenantes en tant que participants au projet.

3. C'est-à-dire freelance, auto-entrepreneur, libéral, entrepreneur salarié ou salarié intermittent.

4. Créée en 1998, cette organisation de l'économie sociale est spécialisée dans les prestations de services et de conseil aux professionnels des secteurs créatifs. Fondée en Belgique (où elle dispose de 11 bureaux), elle a 75000 membres (en 2015) et elle est désormais présente dans 8 pays d'Europe. Elle réalise de nombreuses études en partenariat avec des chercheurs en sciences humaines et sociales. 
Ce travail au projet est proche des modes de fonctionnement de la «cité au projet» proposé par Bolstanki et Chiapello (1999). Ces travailleurs conjuguent activités créatives et/ou artistiques avec des activités secondaires plus ou moins proches de leur savoir-faire principal, pour développer leurs sources de revenus. Cette multiplication des activités participe également d'une volonté de se professionnaliser, d'intégrer le secteur mais aussi de gérer leur carrière. L'artiste est ainsi de plus en plus engagé dans le mode de travail au projet et doit trouver lui-même ses clients. Pour De Heusch et al. (2011, p.17), les travailleurs au projet constituent une figure hybride de travailleurs, qui ne sont ni tout à fait des salariés ni réellement des travailleurs indépendants. Ils sont amenés à gérer eux-mêmes les contrats commerciaux de courte durée liés à leur activité professionnelle. Cette activité est par nature irrégulière et incertaine en passant d'un projet à l'autre au gré des collaborations. Ce travail au projet se traduit par une multi-activité, le recours à un noyau dur (Pilmis, 2007) de clients, la diversification des compétences ainsi que le développement de réseaux professionnels. Comme le souligne P.M. Menger (1991), ces travailleurs sont loin de la figure de «l'artiste bohême» sans attache et libre de tout engagement. Dans la pratique de leurs activités, ces travailleurs au projet cherchent à acquérir et à développer des compétences au fil des projets entrepris, tantôt en termes de créativité, pour aussi mieux maitriser certaines techniques, tantôt dans des domaines connexes, comme la communication, des compétences de gestion pour gérer leur "clientèle», mais aussi pour trouver leurs collaborateurs, leurs commanditaires...

Cette évolution du travail vers le mode projet a des impacts sur le cadre du travail dans la mesure où il requiert une augmentation de la flexibilité, il impose un rythme particulier, il entraîne une disparition de la distinction entre temps de travail et temps privé. Parallèlement, ces travailleurs sont engagés dans des activités non rémunérées - avec l'échange de services, notamment lors des phases de création, d'expérimentation et de répétition aussi. Ces activités contribuent au développement de leur carrière, à la fois pour se faire connaître, étendre leur réseau, mais aussi acquérir de nouvelles compétences.

Travailler au projet, c'est donc mobiliser et mettre en rapport de nombreux intervenants fonctionnant en réseaux (De Heusch et al., 2011). Boltanski et Chiapello (1999, p.157) constatent que «le projet est l'occasion et le prétexte de la connexion (...). La succession de projets, en multipliant les connexions et en faisant proliférer les liens, a pour effet d'étendre les réseaux». Comme le souligne Menger (2005), les réseaux deviennent particulièrement importants dans cette forme de travail organisé par projet, dans la mesure où ils apportent une certaine stabilité aux travailleurs, ils permettent de structurer les rapports entre les individus, dans un environnement où les relations d'emploi sont ponctuelles et variables d'un projet à l'autre. Liot (2010, p.179-180) souligne que le réseau "constitue une structure relativement stable de relations formelles et informelles. Il est traversé à la fois par des pratiques institutionnelles et par des pratiques qui ne s'inscrivent pas dans des relations statutaires». Liot (2010) propose une typologie des différents types de réseau rencontrés dans le secteur artistique entre le réseau local, le réseau marchand et le réseau institutionnel. Cette distinction est reprise par De Heusch et al. (2011, p.26) à propos du réseau local :

Il consiste en une autoproduction des artistes et en une reconnaissance des pairs, importante aux yeux des artistes car elle se substitue à la réussite professionnelle. De plus ce réseau constitue un groupe immédiat de référence et de soutien pour les artistes, en dehors de la concurrence du marché. Sa portée se limite toutefois à la région dans laquelle les artistes mènent leur activité. Ce critère de proximité peut aussi bien être géographique qu'affectifou se poser en termes de valeurs partagées. Dans certains cas, le réseau local peut servir de tremplin vers d'autres réseaux plus porteurs dans la sphère marchande.

Si cette question des réseaux a été explorée ${ }^{5}$, celle du lieu géographique du travail l'est moins. Dans la mesure où, selon Vivant $(2006,2009,2011)$, ces «travailleurs dits créatifs» ont un lien fort à l'espace urbain (au sens de 'place' en anglais), le lieu où ils exercent leur activité ne peut se résumer à des caractéristiques géophysiques. C'est pourquoi les attributs du lieu au sens de la «lieuité» chez Lévy et Lussault (2003, p. 561), c'està-dire "placeness» dans la littérature anglophone font l'objet d'une revue détaillée dans la section suivante.

\section{LA LIEUITÉ : DU LIEU À L'ORGANISATION?}

Précisons d'emblée que si nous nous éloignons délibérément dans cet article du terme «territoire», plus souvent rencontré dans la littérature francophone s'intéressant aux dimensions spatiales des phénomènes organisationnels agglomérés géographiquement, c'est qu'il prend souvent en management stratégique la forme et l'échelle du district, du pôle, du cluster. Il relève alors d'une approche plus économique qu'humaine et sociale des organisations. Or, notre approche se situe ici dans une conception relationnelle (Massey, 2005) et phénoménologique (Casey, 2001), c'est-à-dire plus sensible (Chabaut et al., 2014) de l'espace à l'échelle du travail organisé, espace que nous concevons comme hétérogène, non-isotopique, discontinu et où se mêlent les aspects individuels et collectifs du travail. Cette approche rejoint certaines approches plus psycho-sociales (par ex. Anderson et McAuley (1999), Anderson (2000a, b) ou McKeever et al. (2015) en entrepreneuriat) voire critiques de l'espace-lieu ('place') vécu, construit et se construisant par des appropriations multiples et des relations établies entre acteurs (individus, groupes, collectifs, etc.) et entre acteurs et actants (humains et non-humains) (Sergot et Saives, 2016). Comme l'évoquent Chabault et al. (2014, p. 536), selon cette perspective, «il s'agit alors moins de cartographier des étendues-territoires [délimitées et en extension, nous ajoutons], que de vivre des lieux [en profondeur, nous ajoutons] et des liens de territorialités». C'est pourquoi nous avons choisi de ne pas aborder notre objet (le lieu-quartier) par le biais de la littérature classique en management stratégique sur les territoires, leur développement, leur gouvernance et l'extension de leurs frontières mais d'entrer plutôt dans une littérature à laquelle nous invitent des approches récentes en organisation (Clegg et Van Iterson, 2013) par le biais de l'espace-lieu ('place'). Sans les renier et à des fins de découverte heuristique, nous tentons de nous émanciper 
des variables descriptives classiques des formes territoriales de méta-organisation (les districts, clusters, milieux, pôles, réseaux locaux-globaux) que sont les couples proximité/distance et ouverture/fermeture. Au-delà des approches classiques en stratégie du territoire-lieu centrifuge/central de proximités (Boschma, 2005; Rallet et Torre, 2004), nous abordons l'espace géographique de l'entreprise/de l'entreprendre dans une perspective relationnelle, empruntée à la géographie humaine, sociale et critique, selon laquelle le lieu est construction-distribution hétérogène de phénomènes co-situés dont l'ouverture est consubstantielle de l'existence et dont l'existence est consubstantielle d'appropriations socio-matérielles multiples.

Dans le cadre de cet article, il est cependant impossible de tenter une synthèse des travaux des géographes ${ }^{6}$ sur la notion du lieu tant elle recouvre des acceptions différentes selon les écoles de pensée (cf. la série des ouvrages de Paquot, Younès, et Lussault sur les notions de Territoire (2010), Habiter (2007), Espace et Lieu (2012)). Nous avons choisi de retenir plusieurs propositions faisant consensus et de nous appuyer sur les travaux de Guthey, Whiteman et Elmes (2014) et leur modélisation très pédagogique de la manière dont on peut concevoir le lieu à partir d'une revue de la littérature en géographie relationnelle et sociale notamment. La figure 1 ci-après s'en veut une représentation schématique.

\section{Première proposition, le lieu n'est pas qu'un site:}

Depuis longtemps, les géographes oscillent entre deux conceptions du lieu : tantôt abstraction scientifique mesurable, tantôt réalité sensible de l'écoumène (Berque, p. 555, dans Lévy et Lussault, 2003). Selon Augustin Berque (2012), ces deux conceptions sont sans doute héritées de deux fondements épistémiques à la fois complémentaires et contradictoires empruntés à Platon et Aristote qui, ayant pensé l'espace et le lieu établissent une distinction entre le "topos» et la "chôra". Le topos renvoie au «où» au lieu physique où est situé un corps (indissociable de l'espace qu'il occupe). La chôra renverrait pour sa part au questionnement plus ontologique du «pourquoi» de ce « où». Comme l'explique Berque (2003, p. 555) :

À partir de ces fondements épistémiques se sont déployées deux conceptions possibles du lieu, entre lesquelles oscille encore et toujours la géographie:

- Dans l'une, le lieu est parfaitement définissable en lui-même indépendamment des choses. C'est le lieu des coordonnées cartésiennes du cartographe, dont l'ordonnée (la longitude), l'abscisse (la latitude), et la cote (l'altitude) s'établissent dans l'espace absolu des Principia mathematica de Newton (...)

- l'autre conception possible relève de la chôra. C'est la plus problématique, car elle est essentiellement relationnelle. Le lieu y dépend des choses, les choses en dépendent, et ce rapport est en devenir; il échappe au principe d'identité.

C'est sans doute dans la richesse du concept de la "chôra" que se trouve par exemple le gisement des significations attribuées au lieu ("attachement au lieu») ou le «sens du lieu» ('sense of place' $)^{7}$ aujourd'hui largement discutés dans la littérature récente en géographie. Ainsi, selon Guthey et al. (2014), s'inspirant des travaux d'Agnew (1987), le concept de lieu (ou plus précisément la qualité d'être un lieu : placeness) recouvre aujourd'hui trois dimensions processuelles :

- La localisation (le site) géographique : à la fois géo-position selon des coordonnées spatiales, une topographie, et articulation locale et globale des écosystèmes naturels.

- Le local : défini comme la série de relations informelles et institutionnelles dans laquelle une «place» est située; il comprend à la fois l'histoire, la politique, la culture, l'économie, la communauté, les organisations locales...

- Le 'Sense of place' : qui recouvre un palimpseste et un tissu d'interprétations, d'émotions et de significations. C'est l'environnement construit et la «structure des sentiments" ou encore l'encastrement sensoriel/sensible/sémiotique ${ }^{8}$ des personnes.

On comprend ici que le lieu est plus qu'un seul espace géographique et implique de multiples échelles (individuelles et collectives, sociales et naturelles, locales et globales, etc.) entrecroisées et enchevêtrées, construites au fil de l'expérience du lieu (Herod, et al., 2007) en particulier à l'heure de la mondialisation.

\section{Deuxième proposition : le lieu n'est pas un territoire "figé"}

Selon Guthey et al. (2014, p. 257), les géographes conçoivent donc le lieu comme un processus d'interaction continuelle entre les différents facteurs précédents plutôt que comme un canevas préformé où la vie sociale, économique et organisationnelle se déroulerait.

Les individus forgeant cette vie locale sont-ils néanmoins dans une dynamique de sédentarisation, de constitution d'un territoire construit et se figeant dans l'authenticité, l'homogénéité et des frontières délimitées? Il s’agit ici de repenser l'habiter (appropriation du lieu). Les individus s'approprient certes un espace/ lieu (de façon physique, langagière et idéelle) autant que l'espace/ le lieu influence leur façon de l'habiter (aux différentes échelles de la vie privée et publique). En ce sens, le territoire comme lieu qui rassemble les infinis de l'Être et de l'étant humain, est à la fois mouvement et passage (Dulau, 2009). Le lieu est donc un espace pluriel en constante reconfiguration que nous habitons momentanément individuellement et collectivement autant qu'il nous habite au-delà des seuls murs de la maison (habitation). L'habitabilité des lieux pour tous renvoie alors à un questionnement sur l'hétérogénéité mouvante constitutive du lieu et sur l'ouverture plutôt que sur la fermeture des possibles du lieu.

\section{Troisième proposition : le lieu est une construction relationnelle hétérogène et sérendipitaire (c'est-à-dire source de heureux hasards) :}

Les rencontres fortuites vont permettre de construire un lieu vivant, mouvant et plein de promesses. Dans ce foisonnement de relations entre les humains et les choses, il existe autant d'individus que de façon d'habiter comme autant de rapports

6. Travaux qui s'inscrivent dans des «filiations» philosophiques différentes.

7. Voir aussi, et entre autres, les travaux d'H. Lefebvre sur la production de l'espace où l'espace est considéré comme une production sociale articulant à la fois un espace perçu, un espace vécu et un espace conçu.

8. Ici nous entendons le sens au-delà de la seule «orientation» à la fois comme «sensorialité», «sensibilité» et «signification» à la suite de J-P. Boutinet (1990). 


\section{FIGURE 1}

\section{Lieuité, une synthèse des caractéristiques-qualités d'un lieu}

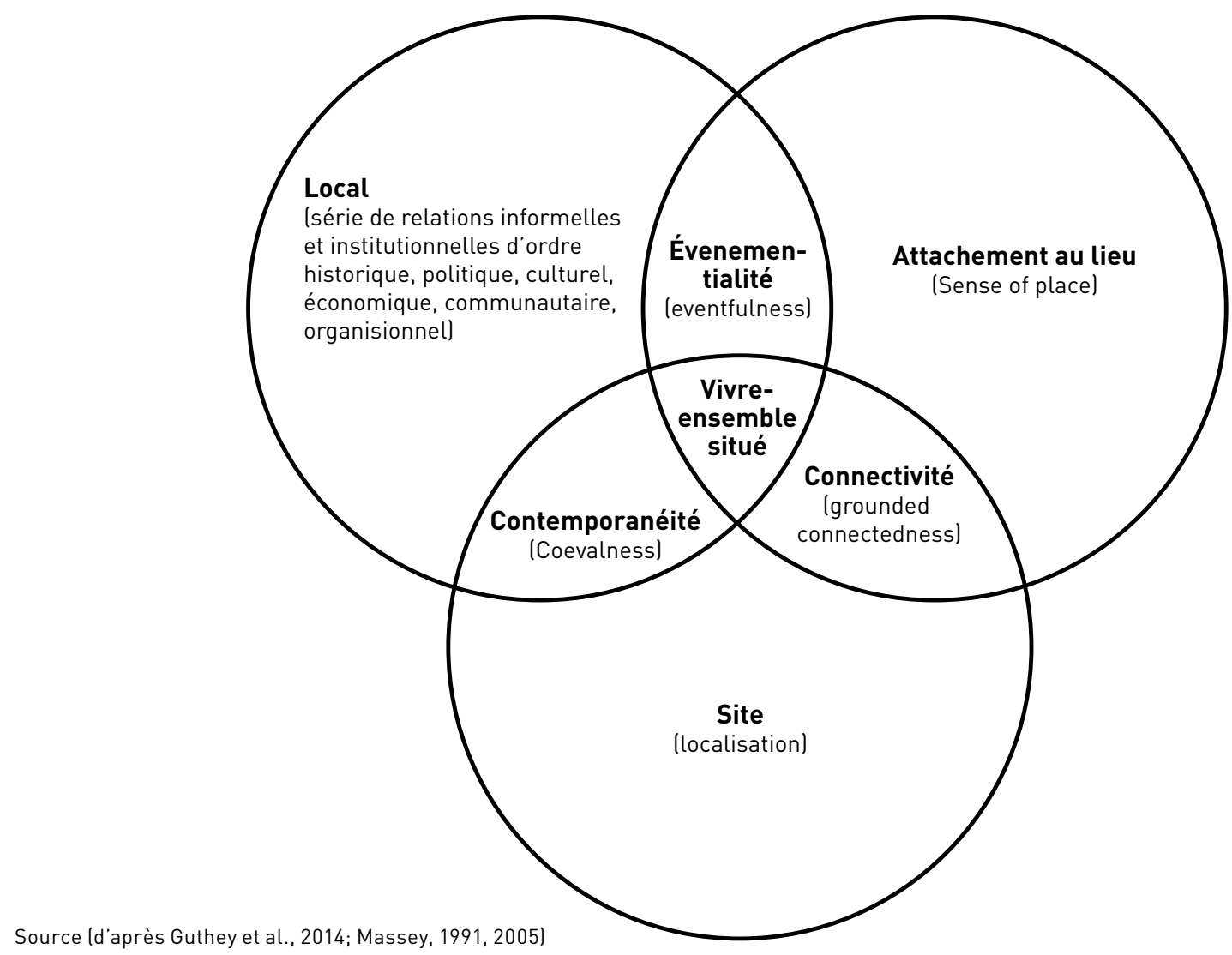

entre soi et le monde. Qui plus est, si le lieu est mouvement et donc dynamique permanente, il est aussi le siège de contradictions et de probables paradoxes inhérents à cette hétérogénéité qui conjugue sans doute à la fois lieu et mobilité, territoire et réseau, station et mouvement (Lévy et Lussault, 2003, p. 441), mais aussi présence et absence, advenu et à venir, etc.

Ces réflexions forgent peut-être une dualité du lieu que l'on retrouve dans deux approches distinctes mais compatibles selon Massey (2005) de la lieuité : la perspective d'une authenticité ancrée (un «là » forgé et homogène), et une perspective plus relationnelle du lieu où se confronte une simultanéité d'hétérogénéités (cf. concept de 'global sense of place', chez Doreen Massey, 2005). Dans cette dernière conception plus relationnelle, politique et dynamique, le lieu est à la fois unique (mais non unitaire), toujours ouvert et inachevé. Il est une production relationnelle, faite d'une multitude de relations sociales et d'une connectivité négociée (grounded connectedness) reliant mutuellement le local et le global, constitutif l'un de l'autre. Le lieu est un lieu de rencontre de moult trajectoires individuelles à la fois humaines et non-humaines très différentes. En raison de leur diversité (de rythme notamment), ces rencontres entre trajectoires peuvent être conflictuelles et appellent une négociation répétée d'un ici-et-maintenant et de leur co-présence en un accord qui fait figure d'événement spatio-temporel ('place-as-event'). Le lieu (la place) a ainsi un caractère d'événementalité (eventfulness). Massey évoque aussi une simultanéité d'hétérogénéités ou une constellation d'histoires-en-cours, mais aussi d'éléments-en-suspens ('loose ends'), bref une simultanéité de co-présences dans la contemporanéité (ou co-évolutivité, en anglais co-evalness) qui donnent au lieu un caractère insaisissable. Le lieu est aussi potentiel de rencontres accidentelles ('chance-of-place') entre trajectoires a priori non-reliées, au-delà de la volonté des personnes concernées. En cela, le lieu est sérendipitaire et productif.

Au total, comme le montre la figure 1, le lieu, à la fois local, localisation (site) et sens-du-lieu, se révèle dans des pratiques matérielles relevant de la négociation d'un vivre ensemble situé ('throwntogetherness') fondé sur la connectivité, la contemporanéité (coevalness) et l'événementialité. Or, des travaux récents nous ouvrent des perspectives sur les convergences entre la lieuité telle que définie ci-dessus et une nouvelle théorisation de l'organisation. Knox et al. (2015) militent ainsi pour une compréhension relationnelle de la spatialisation de l'organisation. Ces évolutions conduisent également à faire sortir la GRH (gestion des ressources humaines) du cadre d'une organisation donnée, et de ses frontières traditionnelles (Cadin, 1997). Aussi, en quoi le lieu ainsi caractérisé est-il vecteur d'intégration et d'implication au travail ? Un détour par la littérature en GRH (gestion des ressources humaines) sur le thème de l'intégration et de la socialisation organisationnelle complètera ici notre cadrage théorique. C'est l'objet de notre prochain point. 


\section{LA SOCIALISATION ORGANISATIONNELLE DES TRAVAILLEURS CRÉATIFS : UN MOYEN DE FAVORISER LEUR IMPLICATION DANS LE LIEU-QUARTIER?}

La socialisation organisationnelle a fait l'objet de nombreux travaux car elle est cruciale pour construire le lien entre l'organisation et le salarié, et plus précisément dans le développement de l'implication au travail des salariés, gage de fidélité et de performance (Allen et Meyer, 1990). La définition du concept de socialisation, issu de plusieurs disciplines (sociologie, psychosociologie, anthropologie) fait aujourd'hui l'objet d'un consensus dans la littérature en gestion : Van Maanen et Schein (1999) la définissent comme «le processus par lequel on enseigne à un individu et par lequel cet individu apprend les ficelles d'un rôle organisationnel». Cette définition a le mérite de souligner les deux acteurs de ce processus : d'une part, l'organisation, d'autre part, l'individu lui-même. Cette dynamique interactive est soulignée par tous les auteurs, de plus en plus nombreux à considérer que c'est dans l'interaction organisation-individu que se construit la relation à l'organisation, par un processus d'apprentissage mutuel (Reichers, 1987). La définition de Van Maanen et Schein sous-entend également la dimension temporelle à l'œuvre dans ce processus. Les travailleurs créatifs, les travailleurs au projet sont emblématiques des bouleversements des formes organisationnelles et à ce titre, soulèvent la question de leur socialisation, envisagée du point de vue d'un individu-entrepreneur dans une organisation qui pourrait être le lieu. C'est en tout cas ce que nous cherchons à mettre en évidence dans ce travail. Aussi allons-nous dans cette présentation mettre l'accent sur les apports de la littérature sur le rôle des individus ${ }^{9}$.

La dynamique temporelle est extrêmement importante. Suivant les auteurs, la socialisation organisationnelle couvre une période de six mois à un an voire deux ans après l'entrée dans l'organisation (Perrot, 2008). Mais s'il est acquis que cette première période est déterminante (elle correspond à ce qu'on appelle la période d'intégration), la socialisation concerne aussi l'étude de différents moments de la carrière. Feldman (1989) ainsi que Chao et al., (1994) encouragent à s'interroger sur le processus de socialisation tout au long de la vie professionnelle. C'est sans doute le cas lorsque des changements importants interviennent (changement de direction, par exemple). Les entrepreneurs de notre enquête sont plutôt récemment arrivés dans le quartier des Olivettes et on peut considérer que l'évolution récente du quartier et de celui proche de l'Ile de Nantes (friche industrielle en reconversion) constituent des modifications contextuelles importantes de nature à constituer un «incident de socialisation» (Schein, 1978). Le processus de socialisation nécessite un apprentissage. Louis (1980) évoque par exemple, l'apprentissage des «valeurs, capacités, comportements attendus et connaissances sociales essentielles pour assumer un rôle organisationnel». On distingue ainsi trois stades de socialisation (Lacaze, 2005) :

- Le stade de la socialisation anticipée : le futur salarié a déjà eu des informations sur l'organisation ou sur son futur emploi (c'est le cas des étudiants en médecine ou encore des informations données lors des entretiens d'embauche),

- Le stade de l'intégration correspond aux premiers moments passés dans l'organisation (la personne s'initie à sa tâche, à la vie de son groupe),

- Le management du rôle, encore appelé changement-acquisition ou métamorphose (le nouvel embauché résout les contradictions de son emploi, qu'elles concernent les relations avec ses collègues ou les incompatibilités avec sa vie privée, comme les horaires).

Le processus de socialisation est appréhendé par quatre "domaines de socialisation" que Fischer (1986) définit comme «ce qui peut être appris et qui est modifié au cours de la socialisation»:

- L'organisation : les objectifs, valeurs et culture, finalement les règles, caractéristiques de l'organisation, culture, aspects informels que le nouvel arrivant doit apprendre.

- Le travail : la maîtrise des tâches, le rôle, les compétences et connaissances requises. Le nouvel embauché mobilise les compétences en fonction de ce que sa tâche requiert. Il peut aussi en développer et acquérir de nouvelles.

- Le groupe de travail : il s'agit de faire connaissance avec les collègues et de nouer des relations d'amitié, de développer des relations de collaboration, des communications, de comprendre les aspects de pouvoir et politiques.

- L'intériorisation des objectifs et valeurs organisationnels qui conduit à un changement identitaire : le nouvel embauché apprend sur lui-même et modifie son comportement. Il adopte une nouvelle façon de se vêtir, il découvre ce que sont ses attentes professionnelles, ses motivations au travail. Il établit ses «ancres de carrière».

Ce sont autour de ces domaines de socialisation que se sont constitués les différents éléments qui façonnent le processus de socialisation. Ils se structurent entre d'une part, les pratiques organisationnelles ${ }^{10}$ et d'autre part, les tactiques individuelles. Les tactiques individuelles recouvrent tous les comportements proactifs que les nouveaux embauchés vont mettre en œuvre. La recherche d'information en est la plus importante : elle est aussi bien d'ordre technique (elle permet de bien remplir le contenu de la mission) que relationnelle (elle permet de s'intégrer parmi les collègues et dans le groupe de travail) qu'organisationnelle (elle concerne la vie de l'organisation). Pour obtenir ces informations, le nouvel embauché doit être actif et il osera d'autant plus l'être que les relations dans le groupe sont bonnes ${ }^{11}$. Les tactiques recouvrent tous les comportements qui permettent d'expérimenter de nouvelles situations et de développer des relations avec les membres de l'organisation.

Pour résumer, nous disposons d'un cadre théorique qui, à l'échelle du quartier étudié interroge la «lieuité» ou encore les caractéristiques du lieu dans une perspective relationnelle ainsi que l'intégration-socialisation et par conséquence l'implication au travail de manière à tenter une réponse à notre question de

9. Pour une revue de littérature complète sur le sujet, on pourra se reporter à l'ouvrage de Delobbe et al. (2005).

10. On y observe des pratiques telles que des formations, la mise en place de tutorat, des réunions, etc.

11. Morrison (1993) in Lacaze, 2005 
recherche : en quoi le lieu (géographique) participe-t-il de l'intégration et de l'implication au travail dans le cas des travailleurs dits créatifs «quasi-solitaires»? Cette question générale recouvre les questionnements ciblés suivants :

- En quoi l'intégration/socialisation au travail est-elle «lieuitaire»?

- Quelles sont les caractéristiques du lieu-quartier ou encore comment définir la «lieuité» d'un quartier pour ce type de travailleur dit créatif?

Dans la section suivante, nous présentons la méthodologie qualitative de recherche déployée pour répondre à ces questions ainsi que le terrain étudié : les travailleurs créatifs du quartier des Olivettes, à Nantes.

\section{Du terrain à l'enquête}

\section{LE QUARTIER DES OLIVETTES, LE CONTEXTE EN QUELQUES LIGNES}

Que savons-nous du lieu-quartier d'implantation des entrepreneurs étudiés? Depuis bientôt vingt années, la ville de Nantes, constituant ici notre terrain plus général d'étude, considère que le développement de son territoire dépendra de sa capacité à concevoir, produire, commercialiser des activités et des biens créatifs et culturels, en favorisant la valorisation de ressources et compétences locales et en en attirant de nouvelles (Charles-Pauvers et al., 2011). A l'instar d'autres grandes villes et communautés urbaines européennes (Scott et Leriche, 2005; Pilati et Tremblay, 2007; O'Connor, 2010; O'Connor et Xin, 2013), cette métropole cherche à accroître son attractivité - tant auprès des professionnels, des gens des milieux culturels et créatifs, des étudiants, des entreprises qu’auprès des secteurs de l'éducation et de la formation- en offrant le meilleur cadre de vie naturel, social et culturel.

Cette ville s'est engagée dans des politiques d'aménagement urbain, avec, dans les années 90 , un premier quartier, un ancien faubourg de la ville qui est ici objet de notre recherche (le quartier des Olivettes, cf. figure 2 pour le situer) puis avec une friche industrielle de près de 330 hectares, autrefois dominée par des activités de l'industrie navale et métallurgique et des activités portuaires (négoce de produits agricoles et bois). Selon des modalités parfois similaires mais aussi spécifiques en raison de l'histoire de ces lieux, ces quartiers ont été investis par des acteurs du champ créatif et culturel plus ou moins avec la complicité (mais aussi sous l'impulsion) des acteurs publics locaux. Ainsi sur ces lieux, depuis une quinzaine d'années, des artistes, de jeunes entreprises des secteurs créatifs et culturels ont été invités à s'implanter voire à se regrouper dans des lieux, des quartiers ou dans des espaces précis, sous l'effet conjugué d'une volonté politique (impulsée par la métropole) et d'engagements des acteurs chargés de l'aménagement de ce territoire urbain; ce processus amorcé depuis une vingtaine d'années a connu une accélération depuis 2009 avec l'institutionnalisation d'un Quartier de la Création (celui de la friche industrielle) alors que la dynamique développée dans l'ancien faubourg où se situe le quartier des Olivettes demeure moins valorisée, du moins dans la communication institutionnelle et dans les médias locaux. Toutefois, notre analyse longitudinale (depuis 2009) nous permet d'apprécier l'ancrage «local ${ }^{12}$ effectif de l'économie créative métropolitaine dans cet ancien faubourg de la ville, objet de profondes transformations dans le cadre d'une ZAC (zone d'aménagement concerté, qui s'est finalisée en 2014). Notre étude porte plus particulièrement sur ce quartier des Olivettes, qui a connu plusieurs vies (activités d'entrée de ville et de commerce maritime et fluvial jusqu'au XIX ${ }^{\mathrm{e}}$, puis industries alimentaires et marché de gros jusqu’aux années 1960) où ses activités se sont délocalisées transformant ce faubourg animé en une zone de friches et d'habitat dégradé (Petiteau, 2012).

Ce faubourg a progressivement évolué avec l'installation de créateurs (artistes, architectes, journalistes, graphistes...) qui ont réinvesti d'abord des maisons abandonnées (des squats négociés ${ }^{13}$ pour certains) puis des nouveaux lieux, redonnant vie aux venelles du quartier et aux ateliers libérés par la relocalisation des activités en périphérie. Voici en quels termes le maire de la ville s'exprimait en 2013 :

Ce quartier a été un laboratoire d'expérimentations: programmation de logement social en centre-ville, réhabilitation des copropriétés, reconversion du patrimoine industriel, accueil d'artistes et de créateurs, concertation autour d'un lieu de présentation du projet, négociation avec les acteurs, transversalité des services, concours d'architecture, mixité urbaine, jardin public de quartier... (in Petiteau, 2012, p.4).

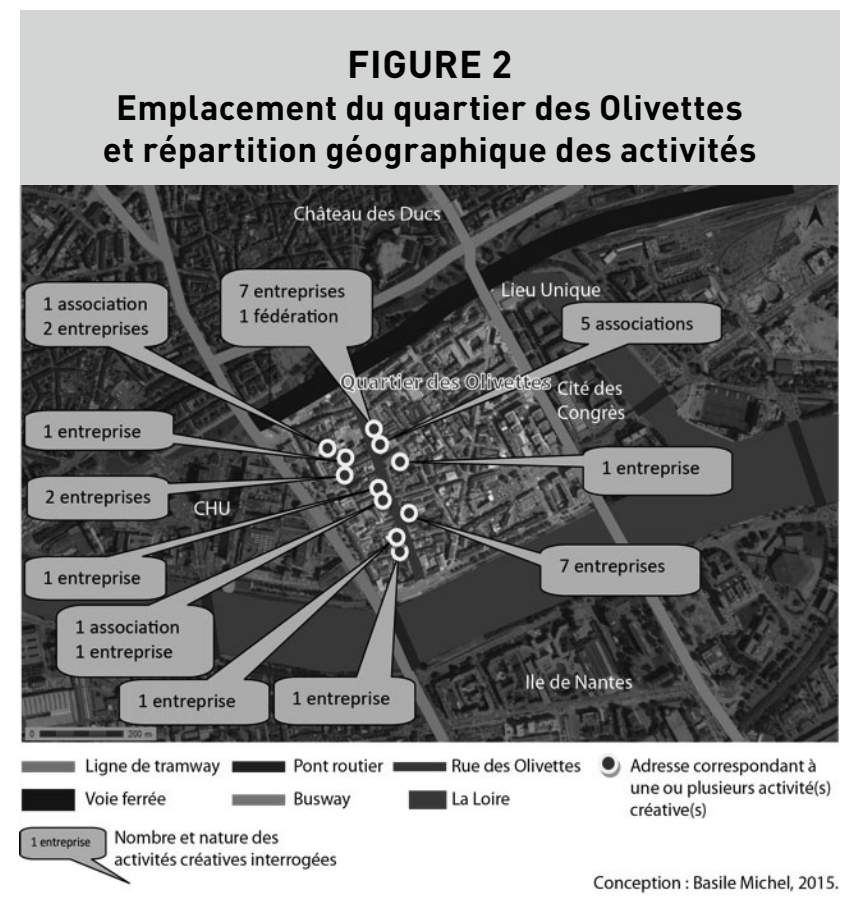

Source. Géoportail

12. (au sens d'une des trois composantes de la lieuité, figure 1)

13. Pour permettre la conservation des locaux désaffectés, éviter les squats incontrôlés et répondre à la demande, la généralisation de contrats à titre gratuit sera engagée avec de jeunes artistes. Selon Petiteau (ibid, p.15): «ce bricolage juridique aura, à terme, un succès considérable sur la valorisation culturelle du quartier. Les jeunes générations seront à l'origine d'un véritable travail de résilience. Madeleine-Champ de Mars devenant pour un temps le quartier de la création culturelle le plus attractif de l'agglomération». 
Ce quartier des Olivettes «s'est développé dans la discrétion sans communication, à tel point que certains parlent aujourd'hui d'un «quartier de la création » qui s'est fait tout seul...» (dans Petiteau, 2012, p.5, propos du maire). Ces premiers éléments descriptifs nous permettent donc de saisir quelques éléments relatifs aux caractéristiques lieuitaires du quartier des Olivettes : une localisation du site géographique du quartier des Olivettes en dehors du Quartier de la Création «officiel»; des relations informelles et institutionnelles locales moins poussées par une démarche politique concertée qu'ailleurs à Nantes. Que disent les entrepreneurs eux-mêmes de leur vie au travail dans ce quartier? C'est l'objet de notre enquête.

\section{DESCRIPTION DE LA MÉTHODOLOGIE D'ENQUÊTE}

Notre étude s'inscrit dans le cadre plus large d'un projet d'étude longitudinale des dynamiques de clusterisation sur le territoire nantais par l'étude de ses principaux lieux (Quartier des Olivettes, Halle Alstom, Quartier de la création, Karting) sur la période 2009-2014 et ceci dans le cadre du programme régional Valeurs et Utilités de la Culture (Pays de la Loire, France). Nous avons choisi de présenter ici les résultats exploratoires d'une enquête menée en 2012 sur le quartier des Olivettes. Notre équipe de recherche a procédé à une série d'entretiens semi-directifs entre avril et octobre 2012 auprès de 46 membres (le plus souvent dirigeants fondateurs, principaux associés ou collaborateurs) d'entreprises ou d'organisations créatives dans le quartier des Olivettes à Nantes. Nous étudions spécifiquement ici le discours de 32 d'entre eux, c'est-à-dire les 32 interlocuteurs rencontrés dans des très petites entreprises de moins de 5 salariés (cf. figure 2), soit la moitié environ du total des organisations créatives du Quartier (cf. figure 3).

L'essentiel des activités rencontrées dans le quartier relèvent des activités dites des industries créatives et culturelles; elles regroupent des designers et graphistes web, des publicistes et spécialistes en agence de communication, des artistes, des architectes, et en plus faible proportion des collectifs d'artistes, des groupements et associations artistiques et culturelles (radio, musique, théâtre, arts plastiques, photographie) ainsi que des galeries d'art. Plus de la moitié des structures de ces entreprises sont le fait de travailleurs indépendants. Près de la moitié des entreprises étudiées n'ont qu'un seul employé, la majorité sont arrivées dans le quartier depuis 2010 seulement, et la dynamique de création d'entreprises dans le quartier est surtout le fait de travailleurs indépendants ces 4 dernières années.

Les entrevues menées autour de questions ouvertes (sur les thématiques suivantes : 1) historique de l'activité et raisons du choix de la localisation, 2) caractéristiques de l'activité, 3) description de leur système relationnel et son évolution, 4) perspectives d'évolution quant à leur activité, leur localisation, leurs relations, les partenariats envisagés, 5) regards sur le quartier et la métropole), d'une durée de 30 minutes à 1 h30 environ, ont été intégralement retranscrites (dans un corpus d'environ 175000 mots).

\section{ANALYSE STATISTIQUE DES RETRANSCRIPTIONS AVeC le logiciel Alceste}

Les retranscriptions ont été soumises à une analyse statistique de contenu textuel à l'aide du logiciel Alceste (Analyse des Lexèmes Co-occurrents dans un Ensemble de Segments de Textes), c'est-à-dire à une (double) classification des discours saillants pour fin de description, de comparaison et d'explication des raisons de l'implantation et des pratiques de travail dans le quartier. Alceste procède en effet à une comparaison systématique deux à deux de morceaux de textes (le corpus est découpé automatiquement en u.c.e $)^{14}$ de façon à établir des classes

\section{FIGURE 3}

Répartition des activités créatives du Quartier des Olivettes selon les secteurs d'activité : comparaison entre l'échantillon enquêté, traité et total

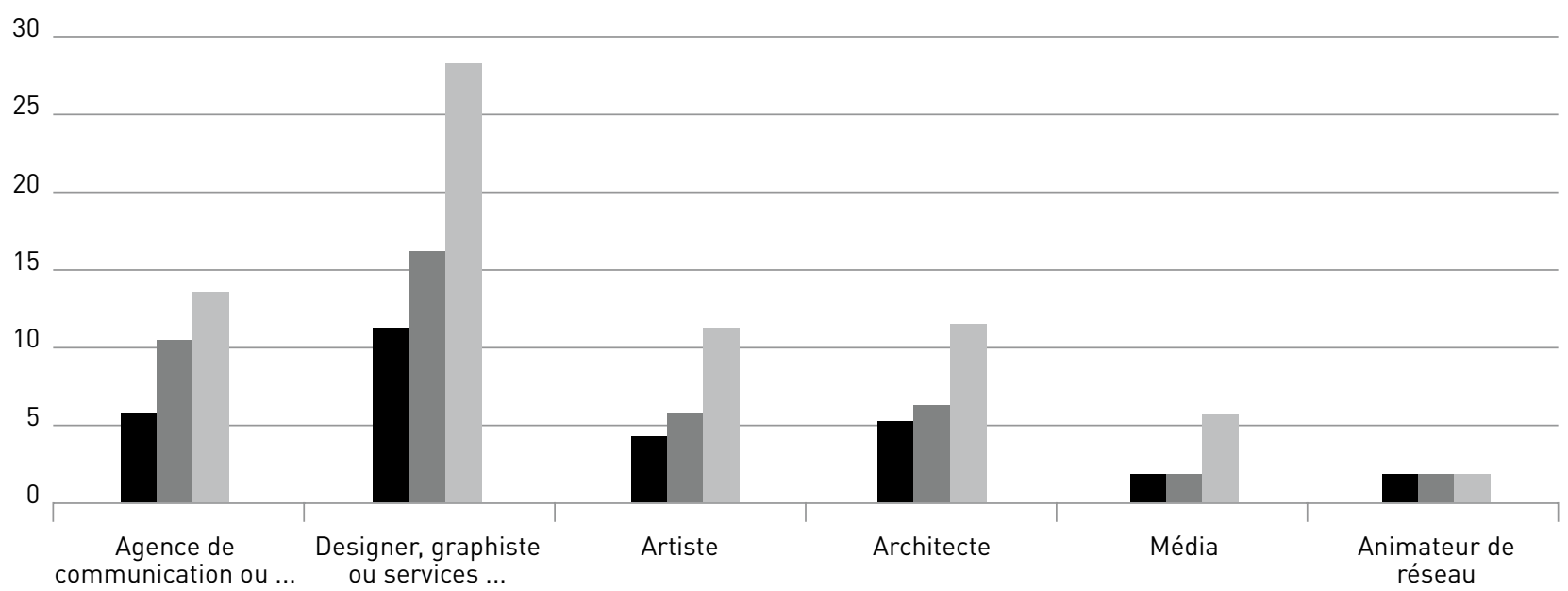


de mots (ou encore ensembles de formes significatives) correspondant à des contenus textuels distincts les uns des autres. Les résultats proposés par le logiciel prennent la forme, entre autres, d'une schématisation de l'arbre de la classification descendante hiérarchique avec les caractéristiques (mots et khi-2 associés) de chacune des classes (cf. figure 4 ci-après) ainsi que d'un inventaire par ordre décroissant des extraits de verbatims les plus emblématiques de ces contextes de discours (contenant les formes significatives précédentes) sur la base d'un Khi-2 d'association significatif dans la classe ${ }^{15}$.

\section{Résultats et discussion : le quartier comme tiers-organisation}

\section{Présentation de la ty Pologie en 3 Classes des DISCOURS}

$81 \%$ des unités textuelles (u.c.e) du corpus des 32 entretiens ont été classées (cf. encadré 1) en 3 groupes de discours après une double classification descendante qui s'avère très stable. La figure 4 ci-après montre la répartition des classes au fil de la double classification et les mots représentatifs de chaque discours distinct.

Le premier niveau de partition distingue d'une part, la classe 1 et d'autre part les classes 2 et 3 . La seconde partition distingue la classe 2 de la classe 3 . La première partition permet de distinguer le cadre opératoire du travailleur créatif (classe 1) du cadre existentiel de son travail (classes 2 et 3). Ensuite, les mondes lexicaux font apparaître l'appropriation de ce cadre par la propriété (classe 3 ) qui se distingue des discours de la classe 2 portant sur une appropriation par les usages du contexte, à savoir celui du quartier.

La classe $1^{16}$ (39\% des u.c.e classées) comprend en effet un monde lexical lié au cadre opératoire du travail créatif. Le vocabulaire significatif de cette classe renvoie d'abord à la forme juridique des activités des travailleurs créatifs, cette forme étant évolutive de la forme associative à d'autres structures (majoritairement indépendantes) :

- Entretien n²1 (F, age_1997, lieu_2003,nb_2) ${ }^{17}$ : «à l'époque, je pense que ça se faisait de fait, dans le secteur culturel et artistique, lorsqu'on voulait monter une structure, c'était une association. C'est encore beaucoup le cas aujourd'hui, même si, depuis, il y a différentes formes juridiques qui sont parfois toutes aussi intéressantes».

- Entretien n 9 (H, age_2011, lieu_2011, nb_1): «ensuite, après une année sous forme associative, on a lancé sous une forme plus sérieuse, donc là, on est à XX... qui est en fait une entreprise qui accompagne les jeunes créateurs donc on est sous forme entrepreneurs salariés, on n'est pas sous forme de SARL».

- Entretien n 12 (F, age_2013, lieu_2011, nb_1) : «je fais du conseil éditorial, de la conception rédaction et des relations presse. Je suis indépendante depuis 2006, je suis passée du statut associatif au statut auto-entrepreneur».

Le vocabulaire de la classe 1 renvoie aussi à différents types d'activités. Parmi les principales activités majoritairement créatives (créer : Khi35=2; idée : Khi42=2), figurent le développement de sites (Khi2=95) et d'activités liées au web (Khi2=36), des activités de graphisme (Khi2=80), de communication (Khi2=53), d'édition $(\mathrm{Khi} 2=26)$ et de design (Khi2=36). Il s'agit d'activités essentiellement connectées (site, web, internet), entrepreneuriales et collaboratives comme en témoignent les termes projet $(\mathrm{Khi} 2=49)$, appeler $(\mathrm{Khi} 2=46)$ et monter (Khi2=54) (associé à des projets, des associations, des structures, des dossiers de subventions, des réponses à des appels d'offre, des événements).

- Entretien n³1 (H, age_2007, lieu_2010,nb_2): «Ca s'appelait $\mathrm{X}$, c'était à $\mathrm{Y}$, enfin c'était une agence de communication, on gérait des bases de données, on faisait des sites internet »

- Entretien n²9 (H, age_2004, lieu_2011, nb_1): «non, il y a eu quelques clients, j'ai fait des journaux dans le sud de la France, un peu dans le nord, des sites internet en sous-traitance d'une entreprise, une boite de communication, mais, en général, c'est dans la région».

- Entretien n 27 (F, age_2011, lieu_2013,nb_1) : «pour la FRAP, j'ai fait du graphisme; j'ai fait une collaboration avec XXX l'année dernière. Après, avec Mr Y qui fait..., qui est directeur artistique évènementiel. J'ai collaboré, j'ai participé à un évènement qu'il a organisé».

Ces travailleurs fonctionnent sur le mode projet (Khi2=49), en réseau, parfois en réponse (répondre: Khi38=2) à des appels d'offre (Khi2 $=41)$ publics ou privés.

- Entretien n³1 (H, age_2007, lieu_2010,nb_2) : «après dans la communication, c'est surtout, aujourd'hui, un travail relationnel. C'est-à-dire qu'on fait travailler un réseau, voilà, donc

14. Les u.c.e (unité de contexte élémentaire) sont des portions de paragraphe comprenant une ou plusieurs phrases, identifiées de façon automatique par le logiciel. Alceste effectue une double classification descendante hiérarchique (CDH) pour regrouper les données textuelles en classes à partir d'un chi-deux d'association qui croise le nombre d'u.c.e. retenues dans le corpus, le nombre d'u.c.e. de la classe considérée, le nombre d'u.c.e. où le mot (dit aussi forme significative) analysé est présent et le nombre d'u.c.e de la classe où le mot (forme significative) est présent.

15. Alceste produit une liste des unités textuelles caractéristiques pour chaque classe, triées par ordre d'importance suivant le Khi2 dans la classe et précédées des variables signalétiques des individus associés. Dans notre analyse, ces variables signalétiques sont : âge (année de fondation de l'entreprise), lieu (année d'implantation dans le quartier des Olivettes étudié) et nb (nombre d'employés dans l'entreprise).

16. Le vocabulaire le plus spécifique de chaque classe est présenté en fonction du Khi2, par ordre de spécificité décroissante. Tous les Khi2 supérieurs à 10.8 sont significatifs au seuil de $0,1 \%$ (le degré de liberté est toujours égal à 1 dans les tableaux de calcul des spécificités avec Alceste). Les mots spécifiques d'une classe seront indiqués en italiques dans le commentaire des différentes classes. Les classes ne sont pas des classes d'individus mais des classes de mots. Le discours de chaque individu peut ainsi traverser les différentes classes.

17. Chaque extrait de verbatim est rapporté à l’individu porteur du discours (cf. note 15 pour la signification des variables signalétiques «Âge», «lieu» et «nb»). 
FIGURE 4

Présentation de la classification des discours en 3 classes

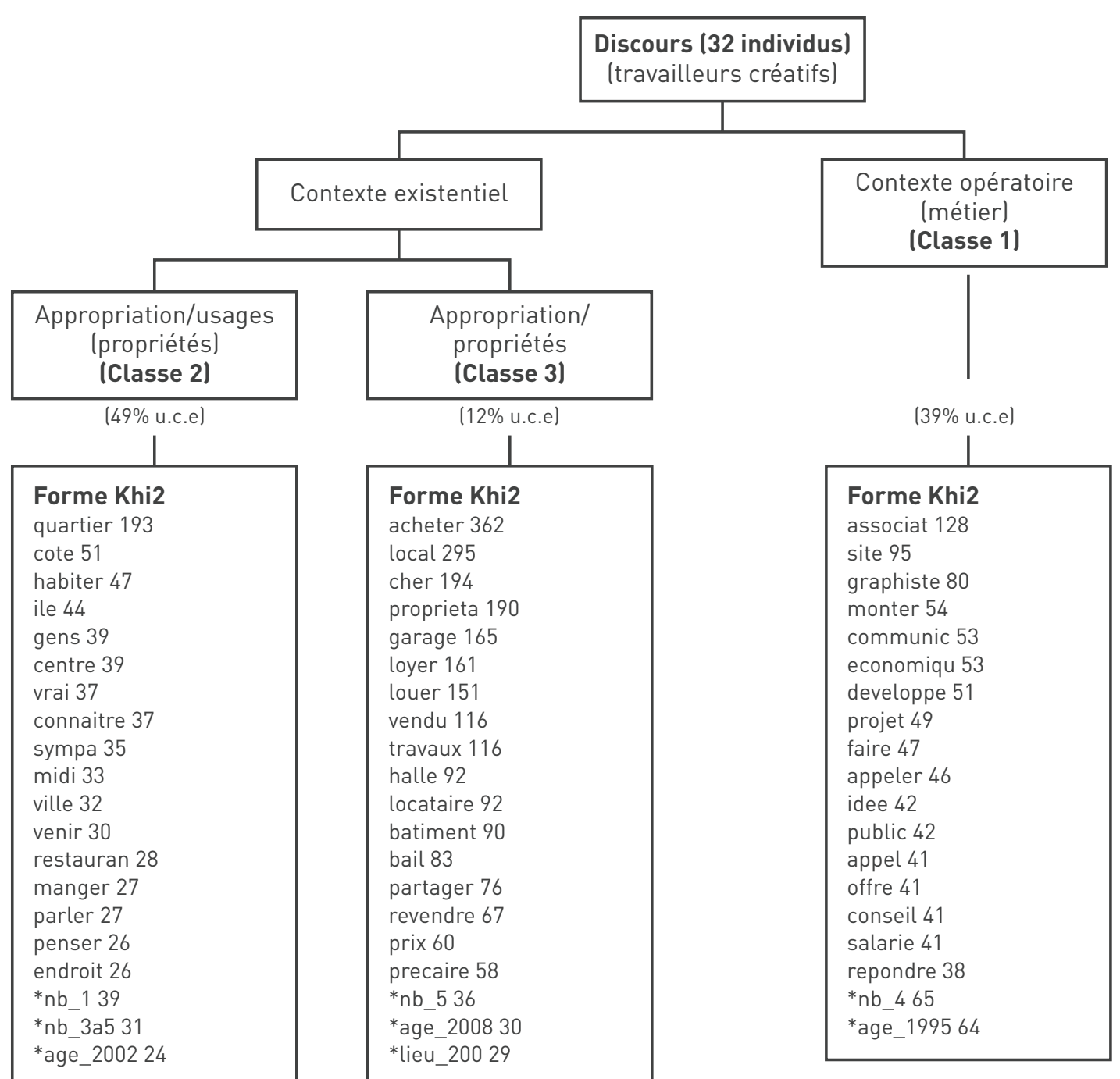

aujourd'hui, c'est dans la communication, hum, on travaille jamais sur appel d'offre, nous, en tout cas».

- Entretien n²2(H, age_2007, lieu_2008,nb_3): «mais bon c'est un..., voilà, c'est... on a fait ce projet là après, on a fait celui de la maison des arts... en signalétique avec un autre architecte qui était XYZ, et voilà, donc, en fait, on a essayé de rentrer en relation ou on est rentrés en relation avec...»

- Entretien n 23 (H, age_2006, lieu_2008,nb_5) : «du coup, on l'a monté comme ça. Après nous, c'est avec les bureaux d'études, les bureaux d'études sur un projet, donc quand on fait un projet, on travaille en équipe, c'est-à-dire qu'on a un bureau d'étude pour la structure, un pour tout ce qui est fluide, c'est-à-dire, l'électricité...".

La classe 3 (12\% des UCE) contient un vocabulaire essentiellement centré sur le cadre existentiel concret du travail créatif (le local (Khi2=295) comme espace de bureaux) et son mode d'appropriation légale : propriété (achat) ou location (acheter (Khi2=362), propriétaire (Khi2=190), louer (Khi2=151)). Il y est question de l'accession à la propriété via l'achat de bonnes affaires, de locaux modestes (garage $:$ Khi2=165) ou vétustes (travaux : Khi116=2, rénover : Khi52=2) :

- Entretien $\mathrm{n}^{\circ} 11(\mathrm{H}$, age_2001, lieu_2007, nb_2) : «il y a un garage là qui s'est vendu. $\mathrm{X}$ a dû acheter son garage 50000 euros en 2005; nous, on l'a perdu, on était déjà à 91000, je crois, c'est quasiment le même prix, pour un garage, c'est rien ».

- Entretien n²3 (H, age_2006, lieu_2008, nb_5) : «c'était quasiment une fixette, il s'en foutait d'attendre, mais il voulait vendre les garages au prix..., je crois que l'histoire, c'est plus ou moins que sa voisine avait vendu un garage à peu près ce prix-là et il ne voulait pas vendre en dessous, voilà. »

- Entretien n $23(\mathrm{H}$, age_2006, lieu_2008, nb_5) : « et puis après, on est arrivés ici..., on s'est installés, en 2008. Alors par contre, je me souviens plus précisément si on s'est installés ici en 2008 ou si on a acheté ici en 2008 , je me souviens plus ».

Il y est aussi question du souci de l'accessibilité financière (cher: khi194=2) des espaces de bureaux (loyers: Khi161=2) que certains partagent (partager, Khi2=76) : 
- Entretien n³1 (H, age_2007, lieu_2010,nb_2): «Ca se loue 180 ici. Et encore, on vaut cher. Là-bas, ça a été vendu très cher, donc ici, ça pas été vendu cher. On avait visité des locaux là-bas, mais on n'avait pas les moyens».

- Entretien n¹9(H,age_2008, lieu_2007,nb_2): «Évidemment, quand nos petits amis du rez-de-chaussée sont partis à côté, on s'est retrouvés à deux, on avait de la place mais on n'avait pas forcément les moyens de payer tout le loyer donc il a fallu trouver des locataires donc on a fait appel à la société d'aménagement qui ont trouvé, voilà».

La classe 2 regroupe le plus grand nombre des u.c.e du corpus (49\%). Le vocabulaire porte lui aussi sur le cadre existentiel des travailleurs mais cette fois au niveau de leur appropriation personnelle de la dynamique du lieu, notamment par les usages du quartier dans lequel ils évoluent. Cinq noyaux distincts de mots ressortent de l'analyse du dendrogramme de la classe :

Un premier noyau autour des mots «quartier» (Khi2=193), «côté» $(\mathrm{Khi} 2=51)$, «ile» $(\mathrm{Khi} 2=44)$ renvoie à une appropriation symbolique (proche du sensemaking (Weick, 1995)) du quartier vu et perçu comme sympa, hybride, mixte, au cachet singulier.

- Entretien n ${ }^{17} 1$ (F, age_2000, lieu_2010, nb_1) : «C'est un quartier sympa à vivre, la rue, là, elle est très bien, on voit les gens passent plutôt à pied ou à vélo donc, c'est ça, c'est assez agréable».

- Entretien nº18 (F, age_2013, lieu_2013,nb_2) : «Après, on était tous emballés quand on a $v u$ les Olivettes, c'est clair qu'il y avait un côté central, en même temps pas trop clean qui nous allait très bien, hein ».

- Entretien n4 (H, age_1997, lieu_2008,nb_3à5) : «Moi, j’adore me balader là-dedans et je trouve ça très chouette, mais voilà, il y a un temps pour tout. Il y a un endroit pour chaque chose et, en tout cas, il était important, que ce soit Ile de Nantes ou ici, que je sois dans le quartier de la création, quoi.»

Un deuxième noyau de mots autour des termes "centre» (Khi39=2), «ville» (Khi32=2), "proximité» (Khi25=2) regroupe des discours sur l'appropriation fonctionnelle du lieu pratiqué comme central, facile, pratique :

- Entretien n³2 (F, age_2004, lieu_2008,nb_1) : «Populaire et tendance, c'est vraiment ça qui me plait ici. Cette espèce de confrontation, de mélange des genres... après, il y a effectivement le fait qu'on soit tout près du centre-ville, les transports en commun, moi, je viens à vélo, quand il ne pleut pas, donc je longe la Loire, c'est hyper-agréable, quoi »

- Entretien n4 (H, age_1997, lieu_2008, nb_3à5) : «Il y a la proximité du centre-ville, évidemment : les transports en commun, la gare, le tramway, le bus-way, la facilité d'accès en vélo, à pied. Ce n'est pas la facilité d'accès en voiture qui m'a fait venir, parce que c'est absolument impénétrable, on ne peut pas stationner, enfin bon sauf le soir, le matin, à des heures bien précises".

Un troisième noyau autour des termes «habiter» (Khi2=47), "connaître» (Khi37=2), "gens» (Khi39=2), "sympas" (Khi35=2) regroupe des discours autour de l'appropriation symbolique sensible du lieu vécu comme vivant, fréquenté et familier, mais dual :
- Entretien n $29(H$, age_2004, lieu_2011, nb_1): «Non, je le pratique peut-être moins [le quartier] que quelqu'un qui habite en plein centre-ville mais non, je vais voir les choses qui s'y passent, je viens en ville quoi».

- Entretien n²1 (F, age_1997, lieu_2003,nb_2) : «Tu connais les petits chemins, tu connais tous les commerces, les petits restaurants de temps en temps, enfin le midi un petit restaurant, surtout chez X.»

- Entretien n²3 (H, age_2006, lieu_2008,nb_5) : «Bah après, quand on est ici, si je passe la journée ici, je vais pas voir forcément, voilà il y a des gens qui se baladent par contre, c'est ça qui est marrant, il y a des gens qui se baladent, on voit clairement, on voit qu'ils remontent la rue».

- Entretien $\mathrm{n}^{\circ} 1$ (H, age_2002, lieu_2003, nb_1): «Non, je pense que ça reste sympa, agréable, sympa, diversifié mais après c'est vrai que, voilà, il y a des deux côtés, hein, c'est un quartier qui reste quand même assez bon..., il y a une part bobo...»

Un quatrième noyau regroupe les termes «midi» $(\mathrm{Khi33}=2)$, «manger» (Khi27=2), "restaurant» $(\mathrm{Khi} 28=2)$, «endroit» (Khi26=2) et évoque la socialisation (réelle ou projetée) dans le quartier, en particulier au moment du midi, dans une ambiance spécifique :

- Entretien n²3 (H, age_2006, lieu_2008, nb_5) : «Donc pour aller manger le midi, il y a plein de restaurants, enfin voilà, il y a plein de petits trucs dans le quartier ».

- Entretien n²9 (H, age_2004, lieu_2011, nb_1) : «Bah, si, si, ça a généré du boulot quand même parce qu'entre bosser chez soi, très peu sortir finalement, et puis travailler dans le centre-ville de Nantes, ça veut dire que le midi, on va manger quelque part, on croise des gens"

- Entretien n4 (H, age_1997, lieu_2008,nb_3à5) : «Je suis arrivé à mon rendez-vous, ça c'est l'endroit qui pour moi est stratégique à Nantes, allez partout comme ça, à la fois avec le 8 et le 10, qu'on a ici et voilà, là, on est au Cul de poule donc la tradition des bars de la rue des Olivettes, c'est quand même, c'est quand même quelque chose et on trouve pas ça partout, hein, avant là où j'étais...»

- Entretien n8 (H, age_2007, lieu_2012,nb_1) : «Non, c'est plutôt j'arrive, je repars. Parce que j'ai une famille, parce que voilà, je suis même plutôt content de rentrer. Mais, sinon, il y a quand même des endroits sympas : le B au bout de la rue, il y a..., on est assez gâté en termes de brasseries, petites brasseries et de choses comme ça, à côté, ce qui est plutôt sympa ne serait-ce que pour avoir des gens au déjeuner»

- Entretien n¹5 (H, age_1982, lieu_2001, nb_4) : «Mais autrement, ça ne devient pas..., on vit bien avec le quartier, on mange aux restaurants, on connait les restaurateurs, et les manifestations culturelles dans le quartier, il y a YY, moi, je vais parfois voir ce qui s'y passe,... mes collègues".

Enfin, un dernier noyau de mots («vrai» (Khi37=2), «vie» (Khi22=2), "penser» (Khi26=2), "rue» $(\mathrm{Khi} 20=2)$ ) évoque plutôt une échelle humaine, et une temporalité synchronique où le rythme urbain coïncide avec celui des travailleurs :

- Entretien n²5 (H, age_1995, lieu_2003,nb_4) : «C'est sympa, on est tout proche du centre-ville, il y a une vraie dynamique 
qui se passe, il y a du 'street art' de partout, il y a plein de gens, on se connait tous, il y a des restaurants cools, je sais pas".

- Entretien n ${ }^{\circ} 1$ (H, age_2002, lieu_2003,nb_1): «Parce que moi, j’appelle vraiment ça le quartier de la Madeleine, ça reste un quartier avec une vraie vie de quartier, un quotidien, avec des choses belles et sympas humainement, parfois beaucoup moins...».

\section{ANALYSE ET Discussion des RÉSUltats}

L'analyse des classes de discours obtenues en lien avec notre revue de littérature nous amène à établir des liens entre la lieuité du quartier nantais étudié et l'implication-intégration au travail des travailleurs créatifs interrogés. En effet, nous décelons plusieurs liens d'intérêt :

- La classe 3, qui comporte essentiellement des éléments relatifs à l'appropriation de l'espace physique de travail, sousentend le caractère souvent récent de l'implantation des travailleurs dans le quartier. En cela, le déménagement ou la création récente de l'entreprise dans le quartier constituent un incident de socialisation qui motive la mise en œuvre de tactiques d'intégration lieuitaires/lieuifiées. L'acte de location ou d'achat peut être interprété en cela comme un acte de fondation (Segaud, 2012) dans/du lieu-organisation en ce que le local du bureau délimite l'espace; il y crée une discontinuité entre espace privé et espace professionnel et oriente cet espace à des fins de travail. Il attache également le travailleur créatif au lieu.

- Les discours sur la structuration juridique des activités des travailleurs créatifs dans la classe 1 (souvent des indépendants, des auto-entrepreneurs) renseignent sur le stade de l'intégration lieuitaire/organisationnelle des individus. Ils montrent des trajectoires de statuts et formes juridiques (structures unipersonnelles, structures associatives, sociétés...) qui rendent compte du processus progressif de socialisation : du stade de la socialisation anticipée à une intégration par la maîtrise du statut juridique de l'activité voire à un management du rôle de l'entrepreneur-créatif qui s'est adapté à sa nouvelle condition de travail. Le travailleur a modifié ses comportements, et intégré les attentes de rôle qu'il a perçues dans ce lieu. Le choix de certains de commencer d'emblée par un statut d'association ou de SARL traduit un entrepreneuriat plus collectif et une intégration au lieu par le biais du groupe d'associés. Les activités décrites par les travailleurs, consistant essentiellement en des projets concourants ou successifs, laissent également penser à une intégration/socialisation du travail toujours à recommencer par le biais de la maîtrise de capacités relationnelles et d'une connectivité (que permet partiellement la concentration des travailleurs dans le quartier) pour travailler en réseau. Dans ce processus, le travailleur-créatif a ainsi modifié ses comportements et ses attitudes pour s'intégrer dans ce lieu.

- Enfin la classe 2 révèle les modes d'appropriation symbolique (l'habiter) de l'espace urbain-quasi-organisation. La socialisation organisationnelle (lieuitaire) passe par :

- La signification de l'image véhiculée par le lieu (dans ce cas-ci, un cachet architectural et «tendance» singulier, un passé ouvrier et populaire qui perdure dans une mixité sociale (diversité), parfois plus observée que vécue) intériorisée par les travailleurs et renforçant l'identité professionnelle collective. Cette image (construction) reste toutefois fragile et en tension.

- Le potentiel de rencontres amicales et professionnelles et les relations sociales que rend possible l'occasion de la socialisation du midi et qui réduit le sentiment d'instabilité des travailleurs, souvent seuls.

- Le sentiment de familiarité et de vitalité nourri par la fréquentation du lieu-quartier qui réduit sans doute le sentiment d'incertitude et de discontinuité du travailleur à projet.

- La centralité de l'espace de travail qui facilite l'appropriation fonctionnelle du lieu et renforce la disponibilité du travailleur flexible.

- L'échelle humaine, le rythme proxémique ressenti par les travailleurs au diapason avec un commun quotidien qui diminue sans doute le sentiment de l'irrégularité et de la variabilité né de la multiplication nécessaire des contrats et des engagements.

Certains éléments de ces résultats sont cohérents avec les observations récentes des géographes en la matière : Chapain et al. (2010) ont en effet observé en Grande-Bretagne des patterns de co-localisation des activités créatives qu'ils attribuent tantôt à des complémentarités sectorielles tantôt à des mouvements intersectoriels de travailleurs créatifs, tantôt à des externalités de connaissances nées du brassage et de l'échange d'informations, d'idées et de pratiques entre professionnels localement. C'est plutôt cette troisième option, à l'échelle de notre quartier qui semble prédominer dans les discours. Même si, nous l'observons chez plusieurs, la socialisation réelle à laquelle renvoient ces échanges se double d'une socialisation rêvée et d'un potentiel souvent encore à explorer. De même, nous retrouvons dans les discours des travailleurs créatifs interrogés une articulation des 4 dimensions inter-reliées qui peuvent déterminer le potentiel de développement d'une économie créative selon Comunian et al. (2010, p. 6) qui ont étudié les micro-interactions entre des travailleurs créatifs et leur contexte local. A l'échelle de notre quartier priment 1) au niveau de la dimension des infrastructures matérielles : la centralité découlant des infrastructures de transport en commun, entre autres, gage de disponibilité, 2) au niveau de la gouvernance : le poids moindre de la gouvernance institutionnelle métropolitaine pour une gouvernance plus autogérée, que reflète partiellement la vitalité du lieu, 3) au niveau des infrastructures intangibles ('soft infrastructures'), l'importance du potentiel de connectivité des réseaux locaux (au sens de Liot, 2010) (ce potentiel pouvant être activé de façon délibérée mais aussi sérendipitaire, c'est-à-dire au hasard de rencontres fortuites), mais surtout d'une identité ou d'une image familière du lieu, ainsi que de traditions et routines qui créent du sens pour les créatifs qui travaillent et vivent ce lieu, traditions et routines au rang desquelles nous situons la quotidienneté ressentie dans le quartier, 4) et enfin au niveau de la dimension des marchés : le primat de la volatilité et de "l'incertitude de la demande» (Caves, 2000) que nous rencontrons dans la généralisation du mode de travail par projet et en réseau(x). 


\section{FIGURE 5 \\ Lieuité et intégration/socialisation organisationnelle des travailleurs créatifs du quartier des Olivettes à Nantes}

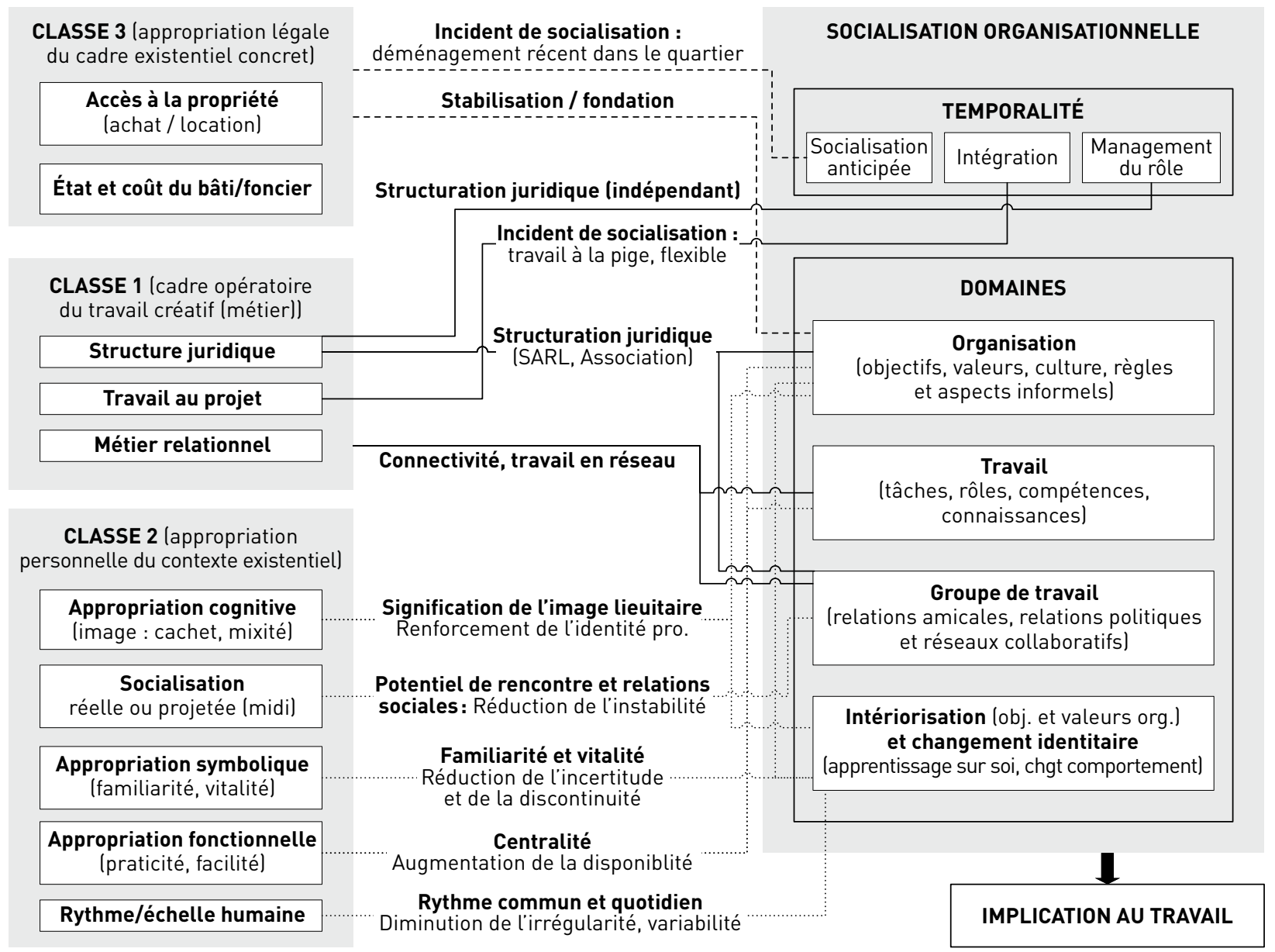

Pour ce qui est de notre champ du management, finalement, en quoi peut-on parler de socialisation organisationnelle des travailleurs dits créatifs? L'approche multidimensionnelle de la socialisation et la dimension temporelle de ce processus nous permettent de mettre en évidence que ces travailleurs créatifs ont mis en place des tactiques individuelles qui les intègrent dans un «lieu » et les font rompre, tout du moins partiellement, avec le caractère individuel propre à leur activité. Ils s'ancrent dans ce lieu-quartier et peu à peu, en modifient le lien juridique, en devenant parfois propriétaires de leur espace de travail. Intégrés dans le quartier, ils y développent des compétences professionnelles et leur travail s'en trouve enrichi : ils travaillent en réseaux et participent à des projets plus complexes. Les entretiens ont largement montré l'influence de ce lieu-quartier sur la constitution de collectifs : opportunités de rencontres amicales et/ ou professionnelles qui peuvent déboucher sur la création de réseaux amicaux mais aussi de réseaux collaboratifs. Au-delà, on sent naître une intériorisation et des changements de comportements liés à l'appropriation fonctionnelle de ce lieu : leur disponibilité augmente et l'irrégularité de leur situation diminue, leur permettant de se réapproprier un quotidien. L'appropriation cognitive et affective de ce lieu construit la socialisation dans ce lieu-organisation. Finalement, ces résultats fournissent un contrepoint aux propos d'Elsa Vivant (2011) qui souligne que pour les travailleurs créatifs, le «corollaire de l'organisation par projet est l'individualisation et la précarisation croissantes qui se traduisent par un travail intermittent et un revenu irrégulier et souvent faible» et que ces travailleurs sont isolés. Le quartier des Olivettes produit une socialisation des travailleurs créatifs qui modifie leurs conditions de travail. Les discours soulignent les modifications du cadre opératoire de leur travail : ils réfléchissent à de nouvelles formes et statuts juridiques, et le lieu favorise les évolutions de leur cadre juridique. Ces travailleurs au projet s'ancrent dans un lieu géographique, à travers un édifice de bureau loué ou acheté parfois. Identité, familiarité et vitalité semblent des caractéristiques lieuitaires propices pour réduire l'incertitude des travailleurs créatifs. De plus, quotidienneté, centralité, connectivité et sérendipité sont des caractéristiques lieuitaires productrices d'opportunités et, en ce sens, réductrices de l'instabilité/irrégularité/discontinuité du travail créatif. 
Ces deux propriétés (réduction de l'incertitude, réduction de l'instabilité) ne renverraient-elles pas à une forme d'inscription de l'activité dans un nouveau cadre organisationnel? Desreumaux (1998), par exemple, rappelle que l'organisation est une réalité qui s'impose en raison des rapports que tout un chacun entretient avec elle (insertion effective ou désirée, confrontation, relations d'échange...). Parmi les conceptions les plus répandues pour caractériser l'organisation par un certain nombre de propriétés structurelles et processuelles, les premières renvoient à l'existence d'un lieu et d'une frontière permettant de distinguer l'organisation d'un univers ou d'un environnement extérieur. Les secondes sont associées à l'intentionnalité et à la capacité à échanger. Le lieu dans son caractère circonscrit (cf. figure 2), autour de la rue des Olivettes et séparé par la Loire et des lignes de tram se distingue d'un autre quartier. Il nous semble que c'est principalement dans l'appropriation personnelle du contexte que le lieu est véritablement source de socialisation. L'habituelle individualisation, l'isolement des travailleurs au projet, voire leurs insécurités (d'ordre existentiel, symbolique, matériel, économique, social, ou psychologique) (Collinson, 2003) sont ainsi partiellement rompus : ce lieu, par ses caractéristiques co-construites par les acteurs qui le traversent et le vivent (centralité, connectivité, quotidienneté, sérendipité d'une part et identité, familiarité, vitalité d'autre part), réduit l'instabilité de leur situation professionnelle et son incertitude. Il est source de temporalisations (par la restauration de la quotidienneté notamment), de relations, de socialisation en même temps que de constructions d'identité. Il se situe donc en réparation de ce que préfigurent beaucoup de situations de travail, - potentiellement néfastes (suicides, burn-out, etc.)—, à l'instar de ce que souligne Menger (2009, 2003, p.6) :

Loin des représentations romantiques, contestataires ou subversives de l'artiste, il faudrait désormais regarder le créateur comme une figure exemplaire du nouveau travailleur, figure à travers laquelle se lisent des transformations aussi décisives que la fragmentation du continent salarial, la poussée

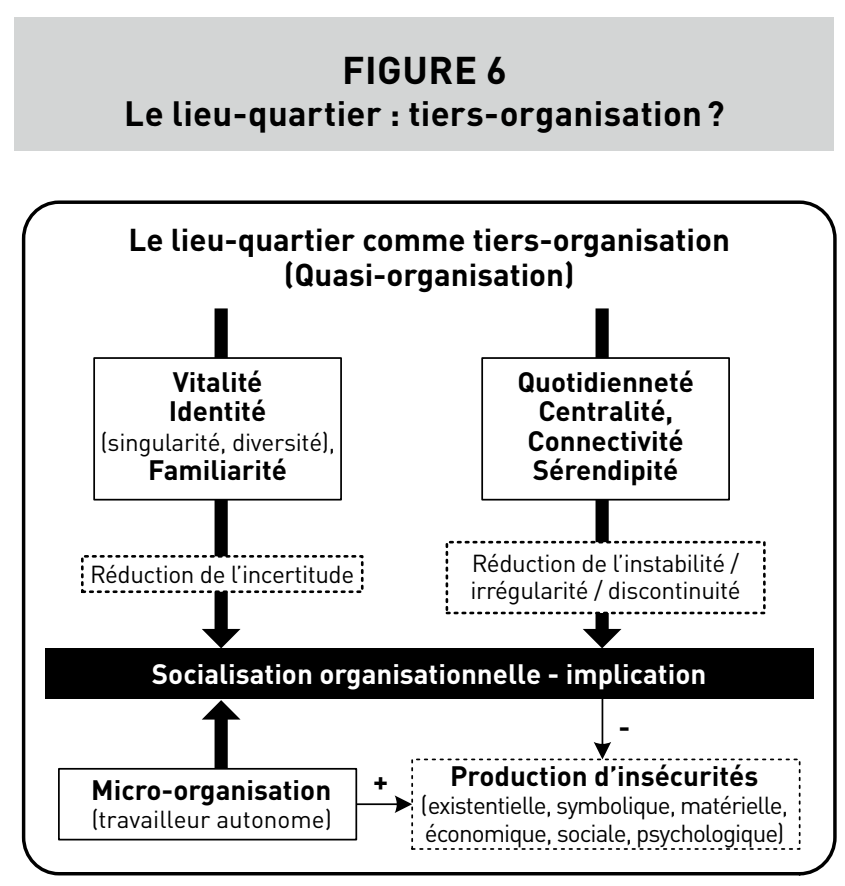

des professionnels autonomes, l'amplitude et les ressorts des inégalités contemporaines, la mesure et l'évaluation des compétences ou encore l'individualisation des relations d'emploi.

\section{Conclusion}

Dans l'esprit des travaux récents qui interrogent la place de l'espace et l'espace de la place en management (Clegg et Kornberger, 2006), notre hypothèse de travail initiale était qu'il nous faut réinvestir le lieu géographique de travail à l'échelle du quartier en tant qu'espace (site) et place (local et sens du lieu) pour le comprendre comme une forme d'organisation spécifique à laquelle se sent appartenir la plupart des travailleurs créatifs en situation de travail autonome. Cette forme d'organisation participe ainsi de leur implication au travail et de la production de sens. Même s'il ne faut pas considérer les travailleurs créatifs étudiés comme une réalité homogène, l'analyse des discours de 32 professionnels du secteur dans le quartier des Olivettes à Nantes fournit un éclairage sur ce lien au lieu en révélant une série de propriétés lieuitaires (identité, familiarité, vitalité, quotidienneté, centralité, connectivité et sérendipité) porteuses d'intégration-socialisation organisationnelle.

En adoptant la conception relationnelle, plurielle, et hétérogène du lieu que nous offrent les travaux récents de géographie relationnelle et sociale, et dans la filiation de travaux comme ceux d'Ahrne et Brunsson (2011) sur l'organisation partielle ('partial organization'), il est peut-être possible de penser le lieu-quartier comme une quasi- ou une tiers-organisation singulière propice à l'intégration car traversée (si l'on reprend les termes de Guattari (2014)) par des «flux» hétérogènes (quotidienneté(s), centralité(s), connectivité(s)), un «machinisme auto-poiétique» (vitalité(s), familiarité(s)), des «valeurs» (identité(s)), et marquée sans doute par la «finitude existentielle» que vient bousculer la fécondité de la sérendipité par exemple. En cela, nous adhérons peut-être à une perspective écosophique du travail, perspective écosophique évoquée par Nicolas Le Strat (2010) et inspirée de Guattari, selon laquelle : «Le travailleur créatif-intellectuel n'agit pas en terrain neutre, mais dans un environnement riche et actif, forcément indéterminé, qui le sollicite dans ses choix, le trouble et le motive, et qu'en retour il sollicitera. Il engage un rapport incisif avec cette multiplicité - une multiplicité à partir de laquelle, avec laquelle, mais aussi contre laquelle, il exerce son talent» (Nicolas-Le Strat, 2010). Encore faut-il que les conditions favorables à cette multiplicité soient réunies : le management des ressources humaines à proposer reste à définir. C'est donc à la compréhension de cette multiplicité que doivent se consacrer nos prochains travaux dans l'approfondissement de cette première étude exploratoire via l'analyse détaillée des séries d'entrevues réalisées depuis 6 ans, à la fois dans le quartier des Olivettes mais aussi dans les différents lieux dits créatifs de Nantes, et ce dans une perspective longitudinale.

En examinant l'articulation lieu/organisation, de tels travaux viendraient également nourrir le débat sur le concept d'organisation relancé aujourd'hui par les propositions d'approches processuelles (l'organisation comme processus-en-cours de devenir ('becoming') (Tsoukas et Chia, 2002)) ou communicationnelles (l'organisation comme gradient d' « organisationalité» chez Dobusch et Schoeneborn (2015)). 
Enfin, au plan théorique, le rapprochement en géographie entre littérature francophone (sur l'objet «territoire») et littérature anglophone (sur l'objet «place») nous semble en soi devoir constituer l'objet d'un article à part entière. Nos interrogations auprès de géographes français d'une part et anglo-américains d'autre part nous montrent que ces deux géographies ne se fréquentent pas. Si les sciences de la gestion sont appelées à créer ce dialogue - ce dont nous sommes convaincus-, cet article n'en était pas le lieu (sans jeu de mots).

\section{Bibliographie}

Aage, t.; Belussi, F. (2008). "From fashion to Design; Creative networks in Industrial Districts", Industry and Innovation, vol. 15, n5, p. 475-491.

Agnew, J. (1987). Place and politics: The geographical mediation of state and society. Boston, MA : Allen \& Unwin.

Ahrne, G.; Brunsson, N. (2011). “Organization outside organizations : the significance of partial organization”, Organization, January, vol. 18, nº 1, p. 83-104.

Allen, N.J.; Meyer, J.P. (1990). “The measurement and antecedents of affective, continuance and normative commitment to the organization", Journal of Occupational Psychology, vol. 63, $\mathrm{n}^{\circ}$, p. 1-18.

Allen, N.J.; Meyer, J.P. (1996). “Affective, Continuance, and Normative Commitment to the Organization : An Examination of Construct Validity", Journal of Vocational Behavior, vol. 49, $\mathrm{n}^{\circ} 3$, p. 252-276.

Ambrosino, C. et Andres, L. (2007). «Régénération culturelle et mutabilité urbaine : un regard franco-britannique» dans F. Leriche (sous la direction de), L'économie culturelle et ses territoires, Toulouse, PUM, p. 305-317.

Ambrosino, C. (2009). «Le cluster culturel, un artefact conceptuel pour mieux comprendre la ville contemporaine» dans C. Vallat et A. Le Blanc, Pérennité urbaine ou la ville au-delà de ses métamorphoses, Traces, Paris, L'Harmattan, p. 59-71.

Anderson, A.R. (2000a). “The protean entrepreneur : the entrepreneurial process as fitting self and circumstance”. Journal of Enterprising Culture, vol. 8, $\mathrm{n}^{\circ} 3$, p. 201-234.

Anderson, A.R. (2000b). "Paradox in the periphery : an entrepreneurial reconstruction”. Entrepreneurship and Regional Development, vol. 12, n² 20, p. 91-109.

Anderson, A.R.; McAuley, A. (1999). "Marketing landscapes : the social context”. Qualitative Market Research, vol. 2, n 3, p. $176-188$.

Andres, L.; Chapain, C. (2013). “The Integration of Cultural and Creative Industries into Local and Regional Development Strategies in Birmingham and Marseille : towards an inclusive and collaborative Governance?", Regional Studies, Feb 1, vol. 47, $\mathrm{n}^{\circ} 2$, p. 161-182.

BAyliss, D. (2007). “Dublin's Digital Hbris : lessons from an Attempt to develop a Creative Industrial Cluster", European Planning Studies; vol. 15, n 9, October, p. 1261-1271.

Berque, A. (2003). «Lieu», dans J. Lévy et M. Lussault (éd.), Dictionnaire de la géographie et de l'espace des sociétés, Paris, Belin, p. 555-556.
Berque, A. (2012). «La chôra chez Platon», chapitre 1 dans T. Paquot et C. Younès (dir.), Espace et lieu dans la pensée occidentale, Paris, La découverte, p. 13-27.

Boltanski, L.; ChiA Pello, E. (1999), Le nouvel esprit du capitalisme, Paris, Gallimard.

Boschma, R.A. (2005). "Proximity and innovation : a critical assessment, Regional Studies, $\mathrm{n}^{\circ} 39$, p. 61-74.

Boutinet, J.-P. (1990). Anthropologie du projet. Paris, PUF.

CADIN, L. (1997). Faut-il sortir la GRH de ses frontières?, in BESSON D., Dedans, dehors, les nouvelles frontières de l'organisation, Paris, Vuibert.

CASEy, E. S. (2001). «Espaces lisses et lieux bruts. L’histoire cachée du lieu», Revue de métaphysique et de morale, 2001/4, $\mathrm{n}^{\circ} 32$, p. 465-481.

Caves, R. E. (2000). Creative industries, Contracts between Art and Commerce, Cambridge, MA : Harvard University Press.

Chabault, D.; Loubaresse, E.; Saives, A-L.; Sergot, B. (2014). «Espaces et territoires», chapitre 40, in F. Tannery, J-P. Denis, T. Hafsi et A-C. Martinet (dir.), Encyclopédie de la stratégie, Paris : Vuibert, p. 533-552.

Chao, G. T.; O’Leary-Kelly, A. M.; Wolf, S.; Klein, H. J. et Gardner, P. D. (1994). "Organizational socialization : Its content and consequences". Journal of Applied Psychology, vol. $79, \mathrm{n}^{\circ} 5$, p. $730-743$.

Chapain, C.; Cooke, P.; De Propris, L.; MacNeill, S.; MateosGARCIA, J. (2010). Creative Clusters and Innovation : putting creativity on the map, NESTA, Research Report, november.

Chapain, C.; Clifton, N.; Comunian, R. (2013). "Understanding Creative Regions : bridging the Gap between Global Discourses and Regional and National Contexts", Regional Studies, Feb 1, vol. $47, \mathrm{n}^{\circ} 2$, p. 131-134.

Charles-Pauvers B.; Schieb-Bienfait N. et Urbain C. (2011). «Unité de lieu / Unité de temps : unité d'action(s) ? De la dynamique de clusterisation : le cas d'une halle regroupant des industries créatives", $X X^{e}$ Conférence de l'AIMS, 7-9 juin, Nantes.

Clegg, S.R.; Kornberger, M. (2006). Space, organizations and management theory, CBS School.

Clegg S.R.; van Iterson, A. (2013), “The effects of liquefying place, time, and organizational boundaries on employee behavior : Lessons of classical sociology", M@n@gement, vol. 16, $\mathrm{n}^{\circ} 5$, p. 621-635.

Clifton, N.; Cooke, P. (2009). "Creative knowledge workers and location in Europe and North America : a comparative review", Creative Industries Journal, vol. 2, $\mathrm{n}^{\circ}$ 1, p. 73-89

Cohendet, P.; Grandadam, D.; Simon, L. (2010). “The Anatomy of the Creative City", Industry and Innovation, vol. 17, $\mathrm{n}^{\circ} 1$, p. 91-111.

Cohendet, P.; Gaffard, J-L. (2012). “Coordination, incitation et création de connaissances”, Management International, vol. 16, numéro spécial, p. 11-19.

Comunian, R.; Chapain, C.; Clifton, N. (2010). "Location, location, location : exploring the complex relationship between creative industries and place", Creative Industries Journal, vol. 3, $\mathrm{n}^{\circ} 1$, p. 5-9. 
Collinson, D.L. (2003). "Identities and Insecurities : Selves at Work”, Organization, vol. 10, n 3, p. 527-547.

Cooke, P.; Lazzeretti, L. (2008). Creative Cities Cultural Clusters and Local Economic Development, Cheltenham/IK, E. Elgar Publishing.

Corsani, A.; M. Lazzarato, (2008). Intermittents et précaires, Éditions Amsterdam.

Cresswell, T. (2007). Place : A short introduction. Malden, MA : Blackwell Publishing.

De Heusch, S.; Dujardin, A. et Rajabaly, H. (2011). «L'artiste entrepreneur, un travailleur au projet», dans le collectif sous la direction du Bureau d'études de SMartBe, L'artiste, un entrepreneur, Les Impressions Nouvelles.

Delobbe, N.; Herrbach, O.; Lacaze, D. et Mignonac K. (2005). Comportement organisationnel (Vol. 1), contrat psychologique, émotions au travail, socialisation organisationnelle, Paris, de Boeck.

DePARTMENT For CUltURE, MEdiA AND SPORTS (DCMS), (1998). Creative Industries Task Force Report. London : DCMS.

DePARTMENT FOR CULTURE, MEdiA AND SPORTS (DCMS), (2001). Creative Industries Mapping Document. London : DCMS. Disponible en ligne à l'adresse : https://www.gov.uk/government/ publications/creative-industries-mapping-documents-2001.

Desreumaux, A. (1998). Théorie des organisations, Paris, EMS

Dobusch, L.; Schoeneborn, D. (2015). «Fluidity, Identity and Organizationality: The Communicative Constitution of Anonymous», Journal of Management Studies, Vol. 52, n 8, December, p. 1005-1035.

Dulau, P. (2009). «Martin Heidegger, la parole et la terre», chapitre 10 dans T. Paquot et C. Younès (dir.), Le territoire des philosophes, Paris, La découverte, p. 177-200.

Feldman, D. (1989). "Socialization, resocialisation, and training”, dans I. Goldstein (ed), Training and development in organizations, San Francisco, Jossey Bass, p. 376-416.

FISHer, C. (1986). "Organizational Socialization : an integrative review", Research in Personnel and Human Resources Management, vol. 4, p. 101-145.

Florida, R. (2002). The Rise of the Creative Class and how it's transforming Work, Leisure, and Everyday Life, New York, Basic Books.

FloridA, R. (2004). Cities and the Creative Class, New York, Routledge.

FlORIDA, R. (2005). The flight of the Creative Class, the New Global Competition for Talent, New-York, Harper Business.

GuatTARI, F. (2014). Qu'est-ce que l'écosophie?, textes présentés par Stéphane Nadaud, Éditions Lignes.

Guthey, G.T.; Whiteman, G.; Elmes, M. (2014). “Place and Sense of Place : Implications for Organizational Studies of Sustainability", Journal of Management Inquiry, vol. 23, n 3, p. 254-265.

Hall P. (2000). "Creative Cities and Economic Development", Urban Studies, vol. $37, \mathrm{n}^{\circ}$ 4, p. 639-649.

Herod, A.; Rainnie, A.; Mcgrath-Champ, S. (2007). "Working space : why incorporating the geographical is central to theorizing work and employment practices", Work, Employment, Society, vol. 21, n 2, p. 247-264.
Hillaire, N. (2011). «Portrait de l'artiste en entrepreneur et de l'entrepreneur en artiste», dans L'artiste un entrepreneur, sous la direction du Bureau d'étude de SMARTBE, Les Impressions Nouvelles.

Klein, H.J.; Molloy, J.C.; Brinsfield, C.T. (2012). "Reconceptualizing workplace commitment to redress a stretched construct : Revisiting assumptions and removing confounds", Academy of Management Review, vol. 37, n 1, p. 130-151.

Klein, H. J.; Cooper, J. T.; Molloy, J. C.; Swanson, J. A. (2014). "The Assessment of Commitment : Advantages of a Unidimensional, Target-Free Approach", Journal of Applied Psychology, vol. 99, n 2, p. 222-238.

Knox, H.; O’Doherty, D.; Vurdubakis, T.; Westrup, C., (2008). "Enacting Airports : Space, Movement and Modes of Ordering", Organization, vol. $15, \mathrm{n}^{\circ} 6$, p. 869-888.

Knox H.; O’Doherty, D.; Vurdubakis, T.; Westrup, C., (2015). "Something happened: Spectres of organization/disorganization at the airport", Human Relations, vol. 68, n6, p. 1001-1020.

LACAzE, D. (2005). «Vers une meilleure compréhension des processus d'intégration : Validation d'un modèle d'intégration proactive des nouveaux salariés ", Revue de Gestion des Ressources Humaines, vol. 56, Avril-Mai-Juin, p. 19-35.

Lefebvre, H. (2000) (4e édition). La production de l'espace, Paris, Economica.

LÉvy, J.; Lussault, M., (2003). Dictionnaire de la géographie et de l'espace des sociétés, Paris, Belin.

Liefooghe, C. (2010). «Economie créative et développement des territoires : enjeux et perspectives de recherche», Innovations, vol. $1, \mathrm{n}^{\circ} 31$, p. 181-197.

Liot, F. (2010). Projet culturel et participation citoyenne. Le rôle de l'animation et de la médiation en question, Paris, L'harmattan.

Louis, M. R. (1980). "Surprise and sense making: What newcomers experience in entering unfamiliar organizational settings". Administrative Science Quarterly, vol. 25, p. 226-251.

Lussault, M., (2009). De la lutte des classes à la lutte des places, Paris, Grasset.

Lussault, M., (2007). L'homme spatial, Paris, Seuil.

Massey, D. (1991). 'A global sense of place', reprinted in Massey, D. (1994) Space, place and gender. Cambridge, Polity Press, p. 146-56.

Massey, D. (2005). For Space, London, Sage.

McKeever, E.; JACK, S.; Anderson, A., (2015). “Embedded entrepreneurship in the creative re-construction of place", Journal of Business Venturing, Vol. 30, $\mathrm{n}^{\circ}$ 1, p. 50-65.

Menger, P.M. (2009). Le travail créateur, s'accomplir dans l'incertain, Paris, Gallimard.

Menger, P.M. (2005). Les intermittents du spectacle. Sociologie du travail flexible, EHESS, coll. "Cas de figure».

Menger, P.M. (2002). Portrait de l'artiste en travailleur, métamorphose du capitalisme, Paris, Seuil.

Menger, P.M. (1991). Marché du travail artistique et socialisation du risqué. Le cas des arts du spectacle, Revue Française de Sociologie, vol. $32, \mathrm{n}^{\circ}$ 1, p. 61-74.

Morrow, P. (1993). The Theory and Measurement of Work Commitment, JAI Press. 
Nicolas-Le Strat, P. (2010). «Le travail créatif-intellectuel : puissance de l'expérimentation et perspectives écosophiques", Récupéré le 19 mars 2014 du site http://www.le-commun.fr/ index.php ?page=le-travail-creatif-intellectuel-puissance-de1-experimentation-et-perspectives-ecosophiques [mis en ligne en juillet 2010]

O'Connor, J.; XIN, G. (2013). “Developing a creative cluster in a post-industrial city : the Creative Industries Development Service (CIDS) and Manchester", In Flew, Terry (Ed.) Creative Industries and Urban Development: Creative Cities in the 21st Century. Routledge (Taylor \& Francis Group), London, p. 43-55.

O'Connor, J., (2010). "The cultural and creative industries : a literature review" [2nd ed.]. Creativity, Culture and Education Series. Creativity, Culture and Education, London.

Paquot, T.; Younès, C. (dir), (2009). Le territoire des philosophes, Paris, La découverte.

PAQuot, T., Younès, C. (dir), (2012). Espace et lieu dans la pensée occidentale, Paris, La découverte.

Paquot, T., Lussault, M., Younès C. (dir). (2007). Habiter, le propre de l'humain, Paris, La découverte.

Petiteau, J.-Y. (2012). Nantes récit d’une traversée MadeleineChamp de Mars, Editions Carré.

Pilati, T., Tremblay, D.G. (2007). «Cité créative et District culturel; une analyse des thèses en présence ", Géographie, Economie et Société, vol. 4, nº 9, p. 381-401.

Pilmis, O., (2007). «Des employeurs multiples au «noyau dur» d'employeurs : relations d'emploi et concurrence sur le marché des comédiens intermittents ", Sociologie du travail, vol. 49, p. 297-315.

Perrot, S., (2008). «Evolution du niveau de socialisation organisationnelle selon l'ancienneté : une analyse des premiers mois dans l'entreprise»,M@n@gement, vol. 11, n³3, p. 231-258

Porter, M., (1998). "Clusters and the New Economics of Competition”, Harvard Business Review, vol. 11, p. 77-90.

Rallet, A.; Torre, A., (2004). "Proximité et localisation», Économie Rurale, vol. 280, nº 1, p. 25-41.

Reichers, A. E., (1987). "An interactionist perspective on newcomer socialization rates". Academy of Management Review, vol. $12, \mathrm{n}^{\circ} 2$, p. $278-287$.

Schein, E., (1978). Career dynamics : Matching Individual and Organizational Needs, Addison-Weysley Publishing Co.

Scotт, A.J., (2010a). "Creative Cities : the role of Culture", Revue d'économie Politique, vol. 120, n 1, p. 181-204.
Scotт, A.J., (2010b). The cultural Economy, London, Sage Publications.

Scott, A.J.; Leriche, F. (2005)., "Les ressorts géographiques de l'économie culturelle : du local au mondial», L'espace géographique, vol. 34, $\mathrm{n}^{\circ} 3$, p. 207-222.

Segaud, M. (2012) (2e édition). Anthropologie de l'espace, Paris, Armand Colin.

Sergot, B.; Saives, A-L., (2016). "Placing organized work : how and why place is a useful concept for Management and Organization Studies", Communication à la $16^{e}$ Conférence EURAM, 2-4 juin, Paris.

SMARTbe (DIR.), (2011). L'artiste, un entrepreneur?, Les Impressions Nouvelles.

Smith, R.; WARfield, K. (2007). "The Creative city : a matter of values", in Cooke P., Lazzeretti L., (eds), Creative cities, Cultural Clusters and Local Economic, Development, E. Elgard, Cheltenham, p. 287-312.

Tremblay, G. (2008). «Industries culturelles, économie créative et société de l'information", Global Media Journal - Canadian edition, vol. $1, \mathrm{n}^{\circ} 1$, p. 65-88.

Tsoukas, H.; CHIA, R., (2002). “On organizational becoming : Rethinking organizational change”, Organization Science, vol. 13, p. 567-582

Turok, I. (2003), “Cities, clusters and Creative Industries : the case of Film and Television in Scotland", European Planning Studies, vol. 11, n 5, p. 549-565.

Van MaAnen, J.; Schein, E. (1999), “Toward a Theory of Organizational Socialization", Research in Organizational Behaviour, vol. 1, p. 209-264.

Vandenberghe, C.; Landry, G.; Panaccio, A-J. (2009). «L'engagement organisationnel», dans. J. Rojot., P. Roussel et C. Vandenberghe (dir.), Comportement organisationnel, volume 3, Bruxelles, De Boeck, p. 275-306.

Vivant, E. (2011). "Travail créatif, emplois précaires", Métropolitiques, 9 novembre 2011. URL : http : //www.metropolitiques.eu/Travail-creatif-emplois-precaires.html.

Vivant, E. (2006). «La classe créative existe-t-elle?», Les annales de la recherche urbaine, $\mathrm{n}^{\circ} 101$, Économies, Connaissances, Territoires, novembre, p. 155-161.

Vivant, E. (2009). Qu'est-ce que la ville créative?, Paris, PUF.

WeICK, K.E. (1995). Sensemaking in organizations. Thousand Oaks, CA : Sage. 